\title{
Morris-Thorne wormholes with a cosmological constant
}

\author{
José P. S. Lemos \\ Department of Physics, Columbia University, \\ New York, NY 10027, \& \\ Centro Multidisciplinar de Astrofísica - CENTRA, \\ Departamento de Física, Instituto Superior Técnico, \\ Av. Rovisco Pais 1, 1049-001 Lisbon \\ e-mail: lemos@physics.columbia.edu \\ Francisco S. N. Lobo \\ Centro de Astronomia e Astrofísica da Universidade de Lisboa, \\ Campo Grande, Ed. C8 1749-016 Lisbon \\ e-mail: flobo@cosmo.fis.fc.ul.pt

\section{Sérgio Quinet de Oliveira} \\ Observatório Nacional - MCT, \\ Rua General José Cristino 77, 20921-400 Rio de Janeiro \\ e-mail: quinet@hotmail.com
}

\begin{abstract}
First, the ideas introduced in the wormhole research field since the work of Morris and Thorne are reviewed, namely, the issues of energy conditions, wormhole construction, stability, time machines and astrophysical signatures. Then, spherically symmetric and static traversable Morris -Thorne wormholes in the presence of a generic cosmological constant $\Lambda$ are analyzed. A matching of an interior solution to the unique exterior vacuum solution is done using directly the Einstein equations. The structure as well as several physical properties and characteristics of traversable wormholes due to the effects of the cosmological term are studied. Interesting equations appear in the process of matching. For instance, one finds that for asymptotically flat and anti-de Sitter spacetimes the surface tangential pressure $\mathcal{P}$ of the thin shell, at the boundary of the interior and exterior solutions, is always strictly positive, whereas for de Sitter spacetime it can take either sign as one could expect, being negative (tension) for relatively high $\Lambda$ and high wormhole radius, positive for relatively high mass and small wormhole radius, and zero in-between. Finally, some specific solutions with $\Lambda$, based on the Morris-Thorne solutions, are provided.
\end{abstract}




\section{Review and Introduction}

It is now 15 years that traversable wormhole theory started in earnest from the work of Mike Morris and Kip Thorne published in 1988 [1]. It was first introduced as a tool for teaching general relativity, as well as an attempt to allure young students into the field, for instance those that had read Contact, a novel of Carl Sagan that uses a wormhole to shortcut a large astronomical distance, but it rapidly spread into several branches. These developments culminated with the publication of the book Lorentzian Wormholes: From Einstein to Hawking by Visser [2], where a review on the subject up to 1995, as well as new ideas are developed and hinted at. It is our intention in this introduction to do a brief review on the subject of wormholes. The subject has grown substantially, and it is now almost out of control. We will focus on the work developed after Visser's book was published (most of the references prior to its releasing are in it), paying attention to the issues that branched out of [1], like the issue of energy conditions, wormhole construction, stability, time machines and astrophysical signatures.

\subsection{The beginning}

It is true that Wheeler [3], before the work of Morris and Thorne [1], had seen wormholes, such as Reissner-Nordström or Kerr wormholes, as objects of the quantum foam connecting different regions of spacetime and operating at the Planck scale, which were transformed later into Euclidean wormholes by Hawking [4] and others, but these Wheeler wormholes were not traversable, one could not cross them from one side to the other and back, and furthermore would, in principle, develop some type of singularity [5]. Having been a student of Wheeler, and having further learned through Wheeler's interaction with Zel'dovich on the trace energy condition (which states $\rho \geq 3 p$, with

$\rho$ being the energy density and $p$ the pressure of the fluid on its rest frame) that energy conditions are on a shaky ground $[6,7]$, Thorne together with his student Morris [1], understood that wormholes, with two mouths and a throat, might be objects of nature, as stars and black holes are. Indeed, it is a basic fact for the construction of traversable wormholes that the null energy condition, the weakest of the conditions, has to be violated. 


\section{$1.2 \quad$ Energy conditions}

The weak energy condition says that the energy density of any system at any point of spacetime for any timelike observer is positive (in the frame of the matter this amounts to $\rho>0$ and $\rho+p \geq 0$ ), and when the observer moves at the speed of the light it has a well defined limit, called the null energy condition $(\rho+p \geq 0)$. The weak and null energy conditions are the weakest of the energy conditions (the null being even weaker than the weak), their violation signals that the other energy conditions are also violated. In Hawking and Ellis' book [8] the weak energy condition is thought a physically reasonable energy condition, that at least all classical systems should obey. Afterwards it was found that it could be violated for quantum systems, such as in the Casimir effect and Hawking evaporation (see [9] for a short review). It was further found that for quantum systems in classical gravitational backgrounds the weak or null energy conditions could only be violated in small amounts, and a violation at a given time through the appearance of a negative energy state, would be overcompensated by the appearance of a positive energy state soon after. This idea gave rise to the averaged energy condition [10], and to the quantum inequalities which, being intermediate between the pointwise energy conditions and the averaged energy conditions, limit the magnitude of the negative energy violations and the time for which they are allowed to exist, yielding information on the distribution of the negative energy density in a finite neighborhood $[11,12,13]$. It seems that the situation has changed drastically, it has been now shown that even classical systems, such as those built from scalar fields non-minimally coupled to gravity, violate all the energy conditions [14] (see also [15] for other violations of the energy conditions). Thus, gradually the weak and null energy conditions, and with it the other energy conditions, might be losing their status of a kind of law.

\subsection{Wormhole construction: a synthesis}

Surely, this has had implications on the construction of wormholes. First, in the original paper [1], Morris and Thorne constructed wormholes by hand, that is, one gives the geometry first, which was chosen as spherically symmetric, and then manufacture the exotic matter accordingly. The engineering work was left to an absurdly advanced civilization, which could manufacture 
such matter and construct these wormholes. Then, once it was understood that quantum effects should enter in the stress-energy tensor, a self-consistent wormhole solution of semiclassical gravity was found [16], presumably obeying the quantum inequalities. These quantum inequalities when applied to wormhole geometries imply that the exotic matter is confined to an extremely thin band of size only slightly larger than the Planck length, in principle preventing traversability [12]. Finally with the realization that nonminimal scalar fields violate the weak energy condition a set of self-consistent classical wormholes was found [17]. It is fair to say that, though outside this mainstream, classical wormholes were found by Homer Ellis back in 1973 [18], and related self-consistent solutions were found by Kirill Bronnikov in 1973 [19], Takeshi Kodama in 1978 [20], and Gérard Clément in 1981 [21], these papers written much before the wormhole boom originated from Morris and Thorne's work [1] (see [22] for a short account of these previous solutions). A self-consistent Ellis wormhole was found again by Harris [23] by solving, through an exotic scalar field, an exercise for students posed in [1].

\subsection{Further wormhole construction}

Traversable wormhole theory achieved the end of its first stage after the writing of the monograph on the subject by Visser in 1995 [2]. This monograph is fairly complete on citations, so we refer the reader to it for a bibliographic search up to 1995. We refer here to some developments afterwards, quoting older references when appropriate.

Further wormhole construction in general relativity:

Visser led the way through several works. Indeed, Visser [24] constructed wormholes with polyhedrical symmetry in 1989, generalized a suggestion of Roman for a configuration with two wormholes [1] into a Roman ring [25], he started a study on generic dynamical traversable wormhole throats [26] in 1997, found classically consistent solutions with scalar fields [14] in 1999, and has also found self-dual solutions [27]. Other authors have made also interesting studies. Before Visser's book we can quote the paper by Frolov and Novikov, where they mix wormhole and black hole physics [28]. After the book, particularly interesting wormholes with toroidal symmetry were found by González-Días [29], wormhole solutions inside cosmic strings were found by Clément [30], and Aros and Zamorano [31], wormholes supported 
by strings by Schein, Aichelburg and Israel, [32], rotating wormholes were found by Teo [33], consistent solutions of the Einstein-Yang-Mills theory in connection with primordial wormhole formation were found in [34], theorems for the impossibility of existence of wormholes in some Einstein-scalar theories were discussed by Saa [35], wormholes with stress-energy tensor of massless neutrinos and other massless fields by Krasnikov [36], wormholes made of a crossflow of dust null streams were discussed by Hayward [37] and Gergely [38], and self consistent charged solutions were found by Bronnikov and Grinyok [39].

Wormhole construction with arbitrarily small violations of the energy conditions:

One of the main areas in wormhole research is to try to avoid as much as possible the violation of the null energy condition. For static wormholes the null energy condition is violated $[1,2]$. Several attempts have been made to overcome somehow this problem: Morris and Thorne already had tried to minimize the violating region in the original paper [1], Visser [24] found solutions where observers can pass the throat without interacting with the exotic matter, which was pushed to the corners, and Kuhfittig [40] has found that the region made of exotic matter can be made arbitrarily small. For dynamic wormholes, the violation of the weak energy condition can be avoided, but the null energy condition, more precisely the averaged null energy condition is not preserved [26, 41, 42], although in [43] it has been found that the quantity of violating matter can be made arbitrarily small, a result in line with [40] for static wormholes.

Wormhole construction with a cosmological constant $\Lambda$ :

Some papers have added a cosmological constant to the wormhole construction. Kim [44] found thin shell solutions in the spirit of Visser [2], Roman [45] found a wormhole solution inflating in time to test whether one could evade the violation of the energy conditions, Delgaty and Mann [46] looked for new wormhole solutions with $\Lambda$, and DeBenedectis and Das [47] found a general class with a cosmological constant. Here, we will further study wormholes in a spacetime with a cosmological constant, as will be detailed below. 
Wormhole construction in other theories of gravitation:

In alternative theories to general relativity wormhole solutions have been worked out. In higher dimensions solutions have been found by Chodos and Detweiler [48], Clément [49], and DeBenedictis and Das [50], in Brans-Dicke theory by Nandi and collaborators [51], in Kaluza-Klein theory by Shen and collaborators [52], in Einstein-Gauss-Bonnet by Kar [53], Anchordoqui and Bergliaffa found a wormhole solution in a brane world scenario [54] further examined by Barceló and Visser [55], and Koyama, Hayward and Kim [56] examined a two-dimensional dilatonic theory.

\subsection{Stability}

To know the stability of an object against several types of perturbation is always an important issue. Wormholes are not an exception. Not many works though are dedicated to the stability theory of wormholes, although the whole formalism developed for relativistic stars and black holes could be readily used in wormholes. Visser [57], Poisson and Visser [58] and Ishak and Lake [59] studied the stability of wormholes made of thin shells and found, in the parameter space $(\mathcal{P} / \Sigma) \times($ throat radius/mass), where $\Sigma$ and $\mathcal{P}$ are, respectively, the surface energy density and surface tangential pressure, those wormholes for which there are stable solutions. For the Ellis' drainhole [18], Armendáriz-Picón [60] finds that it is stable against linear perturbations, whereas Shinkai and Hayward [61] find this same class unstable to nonlinear perturbations. Bronnikov and Grinyok [39,62] found that the consistent wormholes of Barceló and Visser [17] are unstable.

\subsection{Wormholes as time machines}

An important side effect of wormholes is that they can be converted into time machines, by performing a sufficient delay to the time of one mouth in relation to the other. This can be done either by the special relativistic twin paradox method [63] or by the general relativistic redshift way [64]. The importance of wormholes in the study of time machines is that they provide a non-eternal time machine, where closed timelike curves appear to the future of some hypersurface, the chronology horizon (a special case of a Cauchy horizon) which is generated in a compact region in this case. Since time travel to the past is in general unwelcome, it is possible to test whether classical or 
semiclassical effects will destroy the time machine. It is found that classically it can be easily stabilized $[63,2]$. Semiclassically, there are calculations that favor the destruction $[65,66]$, leading to chronology protection [66], others that maintain the machine $[67,25]$. Other simpler systems that simulate a wormhole, such as Misner spacetime which is a species of two-dimensional wormhole, have been studied more thoroughly, with no conclusive answer. For Misner spacetime the debate still goes on, favoring chronology protection [68], disfavoring it [69], and back in favoring [70]. The upshot is that semiclassical calculations will not settle the issue of chronology protection [71], one needs a quantum gravity, as has been foreseen sometime before by Thorne [72].

\subsection{Towards a unified view: From stars to wormholes}

There is now a growing consensus that wormholes are in the same chain of stars and black holes. For instance, González-Días [29] understood that an enormous pressure on the center ultimately meant a negative energy density to open up the tunnel, DeBenedectis and Das [47] mention that the stressenergy supporting the structure consists of an anisotropic brown dwarf 'star', and the wormhole joining one Friedmann-Robertson-Walker universe with Minkowski spacetime or joining two Friedmann-Robertson-Walker universes [26] could be interpreted, after further matchings, as a wormhole joining a collapsing (or expanding) star to Minkowski spacetime or a wormhole joining two dynamical stars, respectively. It has also been recognized, and emphasized by Hayward [73], that wormholes and black holes can be treated in a unified way, the black hole being described by a null outer trapped surface, and the wormhole by a timelike outer trapped surface, this surface being the throat where incoming null rays start to diverge [42, 73]. Thus, it seems there is a continuum of objects from stars to wormholes passing through black holes, where stars are made of normal matter, black holes of vacuum, and wormholes of exotic matter. Although not so appealing perhaps, wormholes could be called exotic stars.

\subsection{Astrophysical signatures}

Stars are common for everyone to see, black holes also inhabit the universe in billions, so one might tentatively guess that wormholes, formed or con- 
structed from one way or another, can also appear in large amounts. If they inhabit the cosmological space, they will produce microlensing effects on point sources at non-cosmological distances [74], as well as at cosmological distances, in this case gamma-ray bursts could be the objects microlensed $[75,76]$. If peculiarly large wormholes will produce macrolensing effects [77].

\subsection{Aim of this paper}

In this paper we extend the Morris-Thorne wormhole solutions [1] by including a cosmological constant $\Lambda$. Morris-Thorne wormholes, with $\Lambda=0$, have two asymptotically flat regions. By adding a positive cosmological constant, $\Lambda>0$, the wormholes have two asymptotically de Sitter regions, and by adding a negative cosmological constant, $\Lambda<0$, the wormholes have two asymptotically anti-de Sitter regions. There are a number of reasons to study wormholes with generic $\Lambda$ that a technologically absurdly advanced civilization might construct. For $\Lambda>0$, we know that an inflationary phase of the ultra-early universe demands it, and moreover, from recent astronomical observations, it seems that we live now in a world with $\Lambda>0$. On the other hand, $\Lambda<0$ is the vacuum state for extended theories of gravitation such as supergravity and superstring theories, and, in addition, even within general relativity, a negative cosmological constant permits solutions of black holes with horizons with topology different from the usual spherical [78, 79] (see [80] for a review), which could be turned into wormhole solutions by adding some exotic matter, although we do not attempt it here.

We follow the spirit of the Morris and Thorne paper [1], in that wormhole theory is a good tool to teach general relativity and a subject that attracts students. We analyze distributions of matter similar to [1] but now with generic $\Lambda$, i.e, we analyze spherically symmetric and static traversable Morris-Thorne wormholes in the presence of a cosmological constant. The more complicated issue of the formalism of junction conditions, that Morris and Thorne so well evaded [1], is here treated also in a pedagogical way through the direct use of Einstein field equation, and the matter content of the thin shell separating the wormhole from the exterior spacetime is found. In this way, an equation connecting the radial tension at the mouth with the tangential surface pressure of the thin shell is derived. The structure as well as several physical properties and characteristics of traversable wormholes due to the effects of the cosmological term are studied. We find that 
for asymptotically flat and anti-de Sitter spacetimes the surface tangential pressure $\mathcal{P}$ of the thin shell is always strictly positive, whereas for de Sitter spacetime it can take either sign as one could expect, being negative (tension) for relatively high $\Lambda$ and high wormhole radius, positive for relatively high mass and small wormhole radius, and zero in-between. Finally, some specific solutions with $\Lambda$, based on the Morris-Thorne solutions, are provided. In presenting these solutions we dwell mostly on the case $\Lambda=0$, and $\Lambda>0$, and comment briefly on $\Lambda<0$. The plan of the paper is as follows: In section 2 we present the Einstein field equation for a wormhole metric and perform the junction to an external asymptotically Minkowski, de Sitter, or anti-de Sitter spacetime. In section 3 we give some wormhole geometries, analogous to [1] having $\Lambda=0, \Lambda>0$ and $\Lambda<0$, and study some of their properties. In section 4 we conclude.

\section{Einstein field equation for wormholes with a generic cosmological constant $\Lambda$}

\subsection{The Einstein field equation with generic $\Lambda$, setting the nomenclature}

To set the nomenclature, the Einstein field equation with a cosmological constant is given, in a coordinate basis, by $\mathrm{G}_{\mu \nu}+\Lambda g_{\mu \nu}=8 \pi G c^{-4} T_{\mu \nu}$, in which $G_{\mu \nu}$ is the Einstein tensor, given by $G_{\mu \nu}=R_{\mu \nu}-\frac{1}{2} g_{\mu \nu} R, R_{\mu \nu}$ is the Ricci tensor, which is defined as a contraction of the Riemann (or curvature) tensor, $R_{\mu \nu}=R_{\mu \alpha \nu}^{\alpha}$, and $R$ is the scalar curvature defined as a contraction of the Ricci tensor, $R=R_{\alpha}^{\alpha}$. The Riemann tensor is a function of the second order derivatives of the metric components $g_{\mu \nu}$. $T_{\mu \nu}$ is the stress-energy tensor of the matter, and $\Lambda$ the cosmological constant [81].

\subsubsection{The spacetime metric}

We will be interested in the spacetime metric, representing a spherically symmetric and static wormhole, given by [1]

$$
d s^{2}=-e^{2 \Phi(r)} c^{2} d t^{2}+\frac{d r^{2}}{1-b(r) / r}+r^{2}\left(d \theta^{2}+\sin ^{2} \theta d \phi^{2}\right),
$$


where $(c t, r, \theta, \phi)$ are the usual spacetime spherical coordinates, and $\Phi(r)$ and $b(r)$ are arbitrary functions of the radial coordinate $r . \Phi(r)$ is designated the redshift function, for it is related to the gravitational redshift, and $b(r)$ is denominated the shape function, because as can be shown by embedding diagrams, it determines the shape of the wormhole [1]. The radial coordinate has a range that increases from a minimum value at $r_{\mathrm{o}}$, corresponding to the wormhole throat, to a maximum $a$ corresponding to the mouth. At $r_{\mathrm{o}}$ one has to join smoothly this spherical volume to another spherical volume copy with $r$ ranging again from $r_{\mathrm{o}}$ to $a$, see Figure 1 . In addition, one has then to join each copy to the external spacetime from $a$ to $\infty$, as will be done.

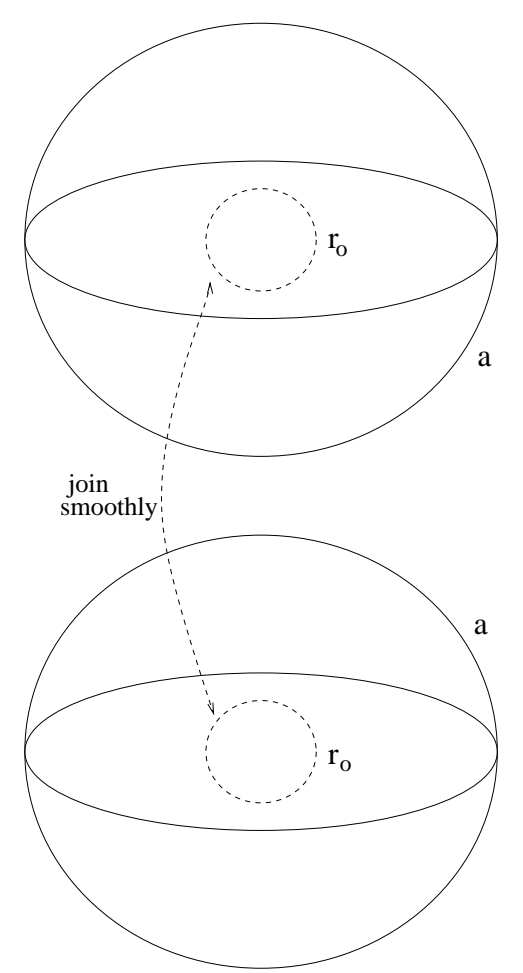

Figure 1 - The two copies of the spherical volume from $r_{\mathrm{o}}$ to $a$ have to be smoothly joined at $r_{\mathrm{o}}$.

The mathematical analysis and the physical interpretation will be simplified using a set of orthonormal basis vectors. These may be interpreted as the proper reference frame of a set of observers who remain at rest in the 
coordinate system $(c t, r, \theta, \phi)$, with $(r, \theta, \phi)$ fixed. Denote the basis vectors in the coordinate system as $\mathbf{e}_{t}, \mathbf{e}_{r}, \mathbf{e}_{\theta}$, and $\mathbf{e}_{\phi}$. Then, using the following transformation, $\mathbf{e}_{\hat{\mu}}=\Lambda_{\hat{\mu}}^{\nu} \mathbf{e}_{\nu}$, with

$$
\Lambda_{\hat{\mu}}^{\nu}=\operatorname{diag}\left[e^{-\Phi},(1-b / r)^{1 / 2}, r^{-1},(r \sin \theta)^{-1}\right] \text {, }
$$

where the notation means that the non-diagonal terms of the matrix are zero, one finds

$$
\left\{\begin{array}{l}
\mathbf{e}_{\hat{t}}=e^{-\Phi} \mathbf{e}_{t} \\
\mathbf{e}_{\hat{r}}=(1-b / r)^{1 / 2} \mathbf{e}_{r} \\
\mathbf{e}_{\hat{\theta}}=r^{-1} \mathbf{e}_{\theta} \\
\mathbf{e}_{\hat{\phi}}=(r \sin \theta)^{-1} \mathbf{e}_{\phi} .
\end{array}\right.
$$

In this basis the metric components assume their Minkowskian form, given by,

$$
g_{\hat{\mu} \hat{\nu}}=\eta_{\hat{\alpha} \hat{\beta}}=\operatorname{diag}(-1,1,1,1) .
$$

In the orthonormal reference frame, the Einstein field equation with a generic cosmological constant, is given by

$$
G_{\hat{\mu} \hat{\nu}}+\Lambda \eta_{\hat{\mu} \hat{\nu}}=\frac{8 \pi G}{c^{4}} T_{\hat{\mu} \hat{\nu}}
$$

The Einstein tensor, given in the orthonormal reference frame by $G_{\hat{\mu} \hat{\nu}}=$ $R_{\hat{\mu} \hat{\nu}}-\frac{1}{2} R g_{\hat{\mu} \hat{\nu}}$, yields for the metric (1) the following non-zero components

$$
\begin{aligned}
G_{\hat{t} \hat{t}} & =\frac{b^{\prime}}{r^{2}} \\
G_{\hat{r} \hat{r}} & =-\frac{b}{r^{3}}+2\left(1-\frac{b}{r}\right) \frac{\Phi^{\prime}}{r}, \\
G_{\hat{\theta} \hat{\theta}} & =\left(1-\frac{b}{r}\right)\left[\Phi^{\prime \prime}+\left(\Phi^{\prime}\right)^{2}-\frac{b^{\prime} r-b}{2 r(r-b)} \Phi^{\prime}-\frac{b^{\prime} r-b}{2 r^{2}(r-b)}+\frac{\Phi^{\prime}}{r}\right], \\
G_{\hat{\phi} \hat{\phi}} & =G_{\hat{\theta} \hat{\theta}},
\end{aligned}
$$

where a prime denotes a derivative with respect to the radial coordinate $r$. 


\subsubsection{The stress-energy tensor}

The Einstein field equation requires that the Einstein tensor be proportional to the stress-energy tensor. In the orthonormal basis the stress-energy tensor, $T_{\hat{\mu} \hat{\nu}}$, must have an identical algebraic structure as the Einstein tensor components, $G_{\hat{\mu} \hat{\nu}}$, i.e., Equations (6)-(9). Therefore, the only non-zero components of $T_{\hat{\mu} \hat{\nu}}$ are $T_{\hat{t} \hat{t}}, T_{\hat{r} \hat{r}}, T_{\hat{\theta} \hat{\theta}}$, and $T_{\hat{\phi} \hat{\phi}}$. These are given an immediate physical interpretation,

$$
\begin{aligned}
T_{\hat{t} \hat{t}} & =\rho(r) c^{2}, \\
T_{\hat{r} \hat{r}} & =-\tau(r), \\
T_{\hat{\theta} \hat{\theta}} & =p(r), \\
T_{\hat{\phi} \hat{\phi}} & =p(r),
\end{aligned}
$$

in which $\rho(r)$ is the energy density, $\tau(r)$ is the radial tension, with $\tau(r)=$ $-p_{r}(r)$, i.e., it is the negative of the radial pressure, $p(r)$ is the pressure measured in the tangential directions, orthogonal to the radial direction. $T_{\hat{\mu} \hat{\nu}}$ may include surface quantities as we will see.

\subsubsection{The cosmological constant and the total stress-energy tensor}

To obtain a physical interpretation of the cosmological constant, one may write the Einstein field equation in the following manner: $G_{\hat{\mu} \hat{\nu}}=8 \pi G c^{-4}\left(T_{\hat{\mu} \hat{\nu}}+\right.$ $\left.T_{\hat{\mu} \hat{\nu}}^{(\mathrm{vac})}\right)$, in which $T_{\hat{\mu} \hat{\nu}}^{(\mathrm{vac})}=-g_{\hat{\mu} \hat{\nu}}\left(\Lambda c^{4} /(8 \pi G)\right)$ may be interpreted as the stressenergy tensor associated with the vacuum, and in the orthonormal reference frame is given by

$$
T_{\hat{\mu} \hat{\nu}}^{(\mathrm{vac})}=\operatorname{diag}\left[\Lambda c^{4} /(8 \pi G),-\Lambda c^{4} /(8 \pi G),-\Lambda c^{4} /(8 \pi G),-\Lambda c^{4} /(8 \pi G)\right] .
$$

We see it is thus possible to adopt the viewpoint that the cosmological term is an integral part of the stress-energy tensor, being considered as a fluid. Accordingly, we can define the total stress-energy tensor, $\bar{T}_{\hat{\mu} \hat{\nu}}$, as

$$
\bar{T}_{\hat{\mu} \hat{\nu}}=T_{\hat{\mu} \hat{\nu}}+T_{\hat{\mu} \hat{\nu}}^{(\mathrm{vac})}
$$

such that $G_{\hat{\mu} \hat{\nu}}=8 \pi G c^{-4} \bar{T}_{\hat{\mu} \hat{\nu}}$. Thus, the components of the total stressenergy tensor of the wormhole, $\bar{\rho}(r), \bar{\tau}(r)$ and $\bar{p}(r)$, are given by the following 
functions

$$
\begin{aligned}
& \bar{\rho}(r)=\rho(r)+\frac{c^{2}}{8 \pi G} \Lambda, \\
& \bar{\tau}(r)=\tau(r)+\frac{c^{4}}{8 \pi G} \Lambda, \\
& \bar{p}(r)=p(r)-\frac{c^{4}}{8 \pi G} \Lambda .
\end{aligned}
$$

This viewpoint may be interesting to adopt in some cases.

\subsubsection{The Einstein equations}

We are interested in matching the interior solution, whose metric is given by Equation (1), to an exterior vacuum solution, which will be considered below. Using Equation (5) and equating Equations (6)-(8) with (10)-(12) we obtain the following set of equations

$$
\begin{gathered}
\rho(r)=\frac{c^{2}}{8 \pi G}\left(\frac{b^{\prime}}{r^{2}}-\Lambda\right) \\
\tau(r)=\frac{c^{4}}{8 \pi G}\left[\frac{b}{r^{3}}-2\left(1-\frac{b}{r}\right) \frac{\Phi^{\prime}}{r}-\Lambda\right] \\
p(r)=\frac{c^{4}}{8 \pi G}\left\{\left(1-\frac{b}{r}\right)\left[\Phi^{\prime \prime}+\left(\Phi^{\prime}\right)^{2}-\frac{b^{\prime} r-b}{2 r^{2}(1-b / r)} \Phi^{\prime}-\frac{b^{\prime} r-b}{2 r^{3}(1-b / r)}+\frac{\Phi^{\prime}}{r}\right]+\Lambda\right\}
\end{gathered}
$$

By taking the derivative of Equation (20) with respect to the radial co-

ordinate $r$, and eliminating $b^{\prime}$ and $\Phi^{\prime \prime}$, given in Equation (19) and Equation (21), respectively, we obtain the following equation

$$
\tau^{\prime}=\left(\rho c^{2}-\tau\right) \Phi^{\prime}-\frac{2}{r}(p+\tau)
$$

Equation (22) is the relativistic Euler equation, or the hydrostatic equation for equilibrium for the material threading the wormhole, and can also be obtained using the conservation of the stress-energy tensor, $T_{; \hat{\nu}}^{\hat{\mu} \hat{\nu}}=0$, putting $\hat{\mu}=r$. The conservation of the stress-energy tensor, in turn can be deduced from the Bianchi identities, which are equivalent to $G_{; \hat{\nu}}^{\hat{\mu} \hat{\nu}}=0$.

\subsubsection{Method for solving the Einstein equations}

The conventional approach to solving the Einstein equations would be to assume a specific and plausible type of matter or fields for the source of the 
stress-energy tensor. One would then derive equations of state for the radial tension and the tangential pressure, as functions of the energy density. These equations of state, together with the three field equations would provide the geometry of the spacetime given in terms of the metric, $g_{\mu \nu}$, as we would have five equations for five unknown functions, i.e., $b(r), \Phi(r), \rho, \tau$ and $p$. Morris and Thorne's approach [1], which will be followed in this paper, differs as they first fixed a convenient geometry for a wormhole solution and then derived the matter distribution for the respective solution (see [47] for a careful analysis of the various approaches).

\subsection{Construction of a wormhole with generic $\Lambda$. I: General comments}

\subsubsection{The mathematics of embedding}

We can use embedding diagrams to represent a wormhole and extract some useful information for the choice of the shape function, $b(r)$, which will be used in the specific solutions considered below. Due to the spherically symmetric nature of the problem, one may consider an equatorial slice, $\theta=\pi / 2$, without loss of generality. The respective line element, considering a fixed moment of time, $t=$ const, is given by

$$
d s^{2}=\frac{d r^{2}}{1-b(r) / r}+r^{2} d \phi^{2} .
$$

To visualize this slice, one embeds this metric into three-dimensional Euclidean space, whose metric can be written in cylindrical coordinates, $(r, \phi, z)$, as

$$
d s^{2}=d z^{2}+d r^{2}+r^{2} d \phi^{2} .
$$

Now, in the three-dimensional Euclidean space the embedded surface has equation $z=z(r)$, and thus the metric of the surface can be written as,

$$
d s^{2}=\left[1+\left(\frac{d z}{d r}\right)^{2}\right] d r^{2}+r^{2} d \phi^{2} .
$$

Comparing Equation (25) with (23) we have the equation for the embedding surface, given by

$$
\frac{d z}{d r}= \pm\left(\frac{r}{b(r)}-1\right)^{-1 / 2}
$$


To be a solution of a wormhole, the geometry has a minimum radius, $r=$ $b(r)=r_{\mathrm{o}}$, denoted as the throat, at which the embedded surface is vertical, i.e., $d z / d r \rightarrow \infty$, see Figure 2. Outside the wormhole, far from the mouth, space can be asymptotically flat, de Sitter, or anti-de Sitter.

(a)

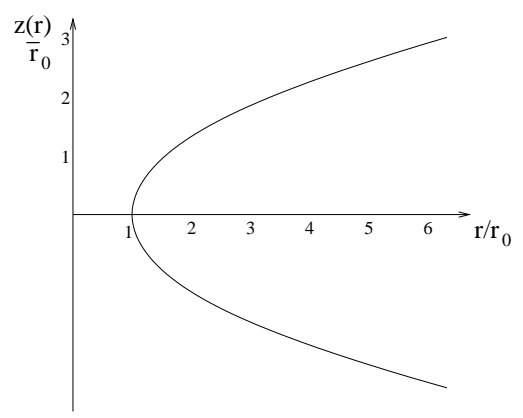

(b)

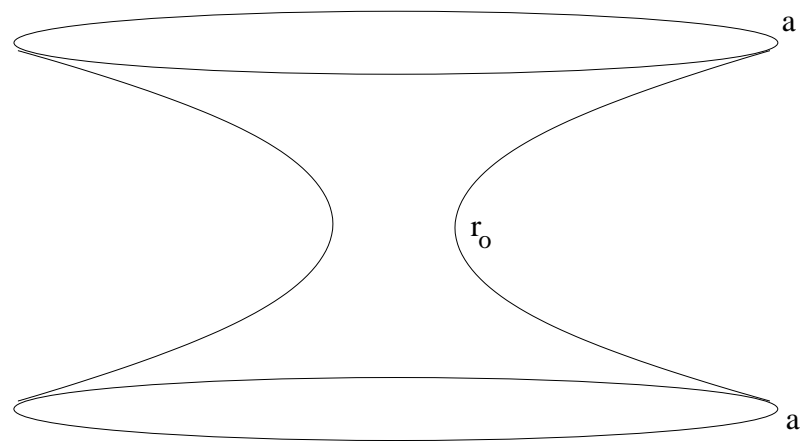

Figure 2 - (a) The embedding diagram of a two-dimensional section $(t=$ constant, $\theta=\pi / 2$ ) of the wormhole in three-dimensional Euclidean space, here $a / r_{\mathrm{o}}=6$; (b) For the full visualization of the surface sweep through a $2 \pi$ rotation around the $z$-axis. Figure 1 with one dimension less is equivalent to this figure.

One can define the proper radial distance for the upper part of the wormhole $z>0$ as

$$
l(r)=\int_{r_{\mathrm{o}}}^{r} \frac{d r}{[1-b(r) / r]^{1 / 2}},
$$

and for the lower part $z<0$ as

$$
l(r)=-\int_{r_{\circ}}^{r} \frac{d r}{[1-b(r) / r]^{1 / 2}} .
$$


The maximum upper limit of integration is $a$, the radius of the wormhole mouth. The shape function $b(r)$ should be positive and such that $b / r<1$ in order to have $\sqrt{r / b-1}$ real. For generic cosmological constant this may not be possible. For instance, in vacuum for sufficiently large radii Equation (26) becomes imaginary, in which case the embedding process is no longer valid. However, the importance of the embedding is near the throat where a special condition, the flare out condition, should be obeyed.

\subsubsection{Exotic matter}

Following [1] closely we will see that the wormhole needs exotic matter, i.e, matter that does not obey the null energy condition, and thus does not obey the weak or any other energy condition. The null energy condition applied to the matter considered in (16)-(17) is $\bar{\rho} c^{2}-\bar{\tau}>0$. Thus a good way to define exoticity is through the parameter $\xi$ defined as $[1] \xi=\frac{\bar{\tau}-\bar{\rho} c^{2}}{\left|\bar{\rho} c^{2}\right|}$. This parameter $\xi$ is dimensionless, and when positive signals exotic matter. Using equations (19)-(20) one finds

$$
\xi=\frac{\bar{\tau}-\bar{\rho} c^{2}}{\left|\bar{\rho} c^{2}\right|}=\frac{b / r-b^{\prime}-2 r(1-b / r) \Phi^{\prime}}{\left|b^{\prime}-\Lambda r^{2}\right|} .
$$

To be a solution of a wormhole, one needs to impose that the throat flares out, as in Figure 2. Mathematically, this flaring-out condition entails that the inverse of the embedding function $r(z)$, must satisfy $d^{2} r / d z^{2}>0$ near the throat $r_{\mathrm{o}}$. Differentiating $d r / d z= \pm(r / b(r)-1)^{1 / 2}$ with respect to $z$, we have

$$
\frac{d^{2} r}{d z^{2}}=\frac{b-b^{\prime} r}{2 b^{2}}>0 \text {. }
$$

Combining Equation (29) with Equation (30), the exoticity function takes the form

$$
\xi=\frac{2 b^{2}}{r\left|b^{\prime}-\Lambda r^{2}\right|} \frac{d^{2} r}{d z^{2}}-2 r\left(1-\frac{b}{r}\right) \frac{\Phi^{\prime}}{\left|b^{\prime}-\Lambda r^{2}\right|} .
$$

Considering the finite character of $\rho$, and therefore of $b^{\prime}$, and the fact that $(1-b / r) \Phi^{\prime} \rightarrow 0$ at the throat, we have the following relationship

$$
\xi\left(r_{\mathrm{o}}\right)=\frac{\bar{\tau}_{0}-\bar{\rho}_{0} c^{2}}{\left|\bar{\rho}_{0} c^{2}\right|}>0 .
$$

Thus matter at the throat is exotic (see $[1,2,26]$ for a detailed discussion). 


\subsection{Construction of a wormhole with generic $\Lambda$. II: Interior and exterior solutions, and junction con- ditions}

We will distinguish the interior cosmological constant, $\Lambda_{\text {int }}$, from the exterior one, $\Lambda_{\text {ext }}$. Equations (19)-(21) demonstrate that once the geometry is fixed by the redshift function $\Phi(r)$, and the shape function $b(r)$, the inclusion of the cosmological constant $\Lambda_{\text {int }}$ will shift the respective values of $\rho(r), \tau(r)$ and $p(r)$ and might help in minimizing the violation of the energy conditions.

\subsubsection{Interior solution of the Einstein equations with generic $\Lambda_{\text {int }}$}

To find an interior solution of the Einstein equations with generic $\Lambda$, we combine the analysis developed in the previous sections, taking into account the notation that $\Lambda_{\text {int }}$ represents the cosmological constant associated with the interior solution. The respective Einstein equations provide the following relationships

$$
\begin{gathered}
\rho(r)=\frac{c^{2}}{8 \pi G}\left(\frac{b^{\prime}}{r^{2}}-\Lambda_{\text {int }}\right) \\
\tau(r)=\frac{c^{4}}{8 \pi G}\left[\frac{b}{r^{3}}-2\left(1-\frac{b}{r}\right) \frac{\Phi^{\prime}}{r}-\Lambda_{\text {int }}\right] \\
p(r)=\frac{c^{4}}{8 \pi G}\left\{\left(1-\frac{b}{r}\right)\left[\Phi^{\prime \prime}+\left(\Phi^{\prime}\right)^{2}-\frac{b^{\prime} r-b}{2 r^{2}(1-b / r)} \Phi^{\prime}-\frac{b^{\prime} r-b}{2 r^{3}(1-b / r)}+\frac{\Phi^{\prime}}{r}\right]+\Lambda_{\text {int }}\right\} \cdot(
\end{gathered}
$$

The metric quantities should carry a subscript int, but we do not put it in order to not overload the notation. It is of interest to find an expression for the radial tension at the throat. From equation (34) one finds that at the throat $\left(b\left(r_{\mathrm{o}}\right)=r_{\mathrm{o}}\right)$ the tension is

$$
\tau\left(r_{\mathrm{o}}\right)=\frac{c^{4}}{8 \pi G}\left(\frac{1}{r_{\mathrm{o}}^{2}}-\Lambda_{\mathrm{int}}\right) .
$$

Thus the radial tension at the throat is positive for wormholes whose structure yields $\Lambda_{\text {int }}<\frac{1}{r_{o}^{2}}$, this includes wormholes with negative and zero cosmological constant. The radial tension is negative, i.e., it is a pressure, for

wormholes with the cosmological constant obeying $\Lambda_{\text {int }}>\frac{1}{r_{0}^{2}}$. The total radial tension, $\bar{\tau}\left(r_{\mathrm{o}}\right)=\tau\left(r_{\mathrm{o}}\right)+\frac{c^{4}}{8 \pi G} \Lambda_{\text {int }}$, is always positive, of course. 


\subsubsection{Exterior vacuum solution of the Einstein equations with generic $\Lambda_{\text {ext }}$}

The spacetime geometry for a vacuum exterior region is simply determined considering a null stress-energy tensor, $T_{\hat{\mu} \hat{\nu}}=0$, i.e., $\rho(r)=\tau(r)=p(r)=$ 0 . Note that $\Lambda_{\text {ext }}$ represents the cosmological constant associated with the exterior solution. In the most general case, the exterior radial coordinate $\bar{r}$, should be different from the interior one $r$. Here we put them equal, both are denoted by $r$, since it simplifies the junction and it gives interestingly enough results. The Einstein equations then reduce to

$$
\begin{gathered}
0=\frac{b^{\prime}}{r^{2}}-\Lambda_{\mathrm{ext}}, \\
0=\frac{b}{r^{3}}-2\left(1-\frac{b}{r}\right) \frac{\Phi^{\prime}}{r}-\Lambda_{\mathrm{ext}}, \\
0=\left(1-\frac{b}{r}\right)\left[\Phi^{\prime \prime}+\left(\Phi^{\prime}\right)^{2}-\frac{b^{\prime} r-b}{2 r^{2}(1-b / r)} \Phi^{\prime}-\frac{b^{\prime} r-b}{2 r^{3}(1-b / r)}+\frac{\Phi^{\prime}}{r}\right]+\Lambda_{\mathrm{ext}} .
\end{gathered}
$$

The metric quantities should carry a subscript ext, but again we have not put it as to not overload the notation. Solving the system of differential equations of Equations (37)-(39), the exterior vacuum solution with a cosmological constant is given by

$$
\begin{gathered}
d s^{2}=-\left(1-\frac{2 G M}{c^{2} r}-\frac{\Lambda_{\mathrm{ext}}}{3} r^{2}\right) c^{2} d t^{2}+\frac{d r^{2}}{\left(1-\frac{2 G M}{c^{2} r}-\frac{\Lambda_{\mathrm{ext}}}{3} r^{2}\right)} \\
+r^{2}\left(d \theta^{2}+\sin ^{2} \theta d \phi^{2}\right) .
\end{gathered}
$$

This metric is the unique solution to the vacuum Einstein equations for a static and spherically symmetric spacetime with a generic cosmological constant. The denomination given to it depends on the sign of $\Lambda_{\text {ext }}$. The Schwarzschild solution, which is a particular case, is obtained by setting $\Lambda_{\text {ext }}=0$. In the presence of a positive cosmological constant, $\Lambda_{\text {ext }}>0$, the solution is designated by the Schwarzschild-de Sitter metric. For $\Lambda_{\text {ext }}<0$, we have the Schwarzschild-anti de Sitter metric. For $\Lambda \neq 0$, note that this metric is not asymptotically flat as $r \rightarrow \infty$, it is either asymptotically de Sitter $\left(\Lambda_{\text {ext }}>0\right)$, or asymptotically anti-de Sitter $\left(\Lambda_{\text {ext }}<0\right)$. However, if $\Lambda_{\text {ext }}$ is extremely small, there is a range of the radial coordinate, i.e., $1 / \sqrt{\Lambda_{\text {ext }}} \gg r \gg G M / c^{2}$, for which the metric is nearly flat. For values of

$r$ below this range, the effect of the mass $M$ dominates, whereas for values above this range, the effect of the cosmological term dominates, as for very 
large values of $r$ the large-scale curvature of the spacetime must be taken into account.

(i) The Schwarzschild spacetime, $\Lambda_{\mathrm{ext}}=0$

Equation (40) with $\Lambda_{\text {ext }}=0$ is the Schwarzschild solution. The full vacuum solution represents a black hole in a asymptotically flat spacetime. The factor $f(r)=\left(1-\frac{2 G M}{c^{2} r}\right)$ is zero at

$$
r_{b}=\frac{2 G M}{c^{2}}
$$

the black hole event horizon. Since the wormhole matter will fill the region up to a radius $a$ larger than $r_{b}$ this radius does not enter into the problem. It is important to have it in mind, since if after construction one finds that $r_{b}>a$ than the object constructed is a black hole rather than a wormhole.

(ii) The Schwarzschild-de Sitter spacetime, $\Lambda_{\mathrm{ext}}>0$

Equation (40) with $\Lambda_{\text {ext }}>0$ represents a black hole in asymptotically de Sitter space. If $0<9 \Lambda_{\text {ext }}\left(G M c^{-2}\right)^{2}<1$, the factor $f(r)=\left(1-\frac{2 G M}{c^{2} r}-\frac{\Lambda_{\text {ext }}}{3} r^{2}\right)$ is zero at two positive values of $r$, corresponding to two real positive roots. Defining

$$
\begin{aligned}
& A=\left(\frac{3 c^{4}}{8 \Lambda_{\mathrm{ext}} G^{2} M^{2}}\right)^{1 / 3} \sqrt[3]{-1+\sqrt{1-\frac{c^{4}}{9 \Lambda_{\mathrm{ext}} G^{2} M^{2}}}}, \\
& B=\left(\frac{3 c^{4}}{8 \Lambda_{\mathrm{ext}} G^{2} M^{2}}\right)^{1 / 3} \sqrt[3]{-1-\sqrt{1-\frac{c^{4}}{9 \Lambda_{\mathrm{ext}} G^{2} M^{2}}}}
\end{aligned}
$$

the solutions are given by

$$
\begin{aligned}
& r_{b}=\frac{2 G M}{c^{2}}\left(-\frac{A+B}{2}-\frac{A-B}{2} \sqrt{-3}\right), \\
& r_{c}=\frac{2 G M}{c^{2}}(A+B) .
\end{aligned}
$$

When $\Lambda_{\text {ext }}\left(G M / c^{2}\right)^{2} \ll 1$ (see appendix A for details), one gets

$$
\begin{aligned}
& r_{b}=\frac{2 G M}{c^{2}}\left[1+\frac{4}{3} \Lambda_{\mathrm{ext}}\left(\frac{G M}{c^{2}}\right)^{2}\right], \\
& r_{c}=\sqrt{\frac{3}{\Lambda_{\mathrm{ext}}}}\left(1-\frac{G M}{c^{2}} \sqrt{\frac{\Lambda_{\mathrm{ext}}}{3}}\right) .
\end{aligned}
$$


The smaller of the values, denoted by $r=r_{b}$, can be considered as the event horizon of the vacuum black hole solution, but since the wormhole matter will fill the region up to a radius $a$ superior than $r_{b}$ this radius does not enter into the problem. The larger value, denoted by $r=r_{c}$, can be regarded as the position of the cosmological event horizon of the de Sitter spacetime. Keeping $\Lambda_{\text {ext }}$ constant, but increasing $M, r=r_{b}$ will increase and $r=r_{c}$ will decrease. If $9 \Lambda_{\text {ext }}\left(G M c^{-2}\right)^{2}=1$, both horizons coincide and are situated at $r=r_{b}=r_{c}=3 G M / c^{2}$. Thus we will consider $9 \Lambda_{\text {ext }}\left(G M c^{-2}\right)^{2}<1$. Particular cases are, $\Lambda_{\text {ext }}=0$ yielding the Schwarzschild solution, and $M=0$ yielding the de Sitter solution. When $r \rightarrow \infty$ the metric tends to the de Sitter spacetime

$$
d s^{2}=-\left(1-\frac{\Lambda_{\text {ext }}}{3} r^{2}\right) c^{2} d t^{2}+\frac{d r^{2}}{\left(1-\frac{\Lambda_{\text {ext }}}{3} r^{2}\right)}+r^{2}\left(d \theta^{2}+\sin ^{2} \theta d \phi^{2}\right) .
$$

For $\Lambda_{\text {ext }} \rightarrow 0$, the de Sitter metric tends to the Minkowskian spacetime. In the coordinates adopted above, the metric of the de Sitter spacetime will be singular if $r=\left(3 / \Lambda_{\mathrm{ext}}\right)^{1 / 2}$, but this is a mere coordinate singularity signaling the presence of a cosmological event horizon.

(iii) The Schwarzschild-anti de Sitter spacetime, $\Lambda_{\mathrm{ext}}<0$

For the Schwarzschild-anti de Sitter metric, with $\Lambda_{\text {ext }}<0$, the equation $f(r)=\left(1-\frac{2 G M}{c^{2} r}+\frac{\left|\Lambda_{\text {ext }}\right|}{3} r^{2}\right)=0$ will have only one real root, therefore implying the existence of one horizon. If one defines

$$
\begin{aligned}
& A=\left(\frac{3 c^{4}}{8\left|\Lambda_{\text {ext }}\right| G^{2} M^{2}}\right)^{1 / 3} \sqrt[3]{1+\sqrt{1+\frac{c^{4}}{9\left|\Lambda_{\text {ext }}\right| G^{2} M^{2}}}}, \\
& B=\left(\frac{3 c^{4}}{8\left|\Lambda_{\text {ext }}\right| G^{2} M^{2}}\right)^{1 / 3} \sqrt[3]{1-\sqrt{1+\frac{c^{4}}{9\left|\Lambda_{\text {ext }}\right| G^{2} M^{2}}}},
\end{aligned}
$$

then the solution is (see appendix for details),

$$
r_{b}=\frac{2 G M}{c^{2}}(A+B) \text {. }
$$

For $\left|\Lambda_{\text {ext }}\right|\left(G M / c^{2}\right)^{2} \ll 1$ one obtains

$$
r_{b}=\frac{2 G M}{c^{2}}\left[1-\frac{4}{3}\left|\Lambda_{\mathrm{ext}}\right|\left(\frac{G M}{c^{2}}\right)^{2}\right] .
$$


Once again this event horizon is avoided by filling the space with exotic matter from the throat at $r_{\mathrm{o}}$ up to the mouth at $a$, where $a>r_{b}$ in order that the wormhole is not a black hole. If $\Lambda_{\text {ext }}=0$, the metric is reduced to the Schwarzschild solution. If $r \rightarrow \infty$ the metric tends to the anti-de Sitter solution

$$
d s^{2}=-\left(1+\frac{\left|\Lambda_{\mathrm{ext}}\right|}{3} r^{2}\right) c^{2} d t^{2}+\frac{d r^{2}}{\left(1+\frac{\mid \Lambda_{\mathrm{ext}}}{3} r^{2}\right)}+r^{2}\left(d \theta^{2}+\sin ^{2} \theta d \phi^{2}\right) .
$$

For $\left|\Lambda_{\text {ext }}\right| \rightarrow 0$, the anti-de Sitter metric tends to the Minkowskian spacetime.

\subsubsection{Junction conditions in wormholes with generic $\Lambda_{\text {ext }}$}

To match the interior to the exterior one needs to apply the junction conditions that follow from the theory of general relativity. One of the conditions imposes the continuity of the metric components, $g_{\mu \nu}$, across a surface, $S$, i.e., $\left.g_{\mu \nu(\mathrm{int})}\right|_{S}=\left.g_{\mu \nu(\mathrm{ext})}\right|_{S}$. This condition is not sufficient to join different spacetimes. One formalism of matching, that leads to no errors in the calculation, uses the extrinsic curvature of $S$ (or second fundamental form of the surface $S$, the first fundamental form being the metric on $S$ ) see, e.g, [82]. However, for spacetimes with a good deal of symmetry, such as spherical symmetry, one can use directly the field equations to make the match, see e.g., [83] (see also Taub [84]). We follow this latter approach. Indeed, due to the high symmetries of the solution, we can use the Einstein equations, Equations (19)-(21), to determine the energy density and stresses of the surface $S$ necessary to have a match between the interior and exterior. If there are no surface stress-energy terms at the surface $S$, the junction is called a boundary surface. If, on the other hand, surface stress-energy terms are present, the junction is called a thin shell.

\section{(i) Matching of the metric}

As was mentioned above the unique vacuum static and spherically symmetric solution, in the presence of a non-vanishing cosmological constant, is given by Equation (40). A wormhole with finite dimensions, in which the matter distribution extends from the throat, $r=r_{\mathrm{o}}$, to a finite distance $r=a$, obeys the condition that the metric is continuous. Due to the spherical symmetry the components $g_{\theta \theta}$ and $g_{\phi \phi}$ are already continuous, and so 
one is left with imposing the continuity of $g_{t t}$ and $g_{r r}$,

$$
\begin{aligned}
& g_{t t(\mathrm{int})}=g_{t t(\mathrm{ext})}, \\
& g_{r r(\mathrm{int})}=g_{r r(\mathrm{ext})},
\end{aligned}
$$

at $r=a$, with $g_{t t(\mathrm{int})}$ and $g_{r r(\mathrm{int})}$ being the metric components for the interior region at $r=a$, and $g_{t t(\mathrm{ext})}$ and $g_{r r(\mathrm{ext})}$ the exterior metric components for the vacuum solution at $r=a$. For the sake of consistency in the notation this could have been done in the orthonormal frame with the hat quantities $g_{\hat{\mu} \hat{\nu}}$, but in this case it is more direct to do with coordinate frame quantities. One can start the analysis by considering two general solutions of Equation (1), an interior solution and an exterior solution matched at a surface, $S$. The continuity of the metric then gives generically $\Phi_{\text {int }}(a)=\Phi_{\text {ext }}(a)$ and $b_{\text {int }}(a)=b_{\text {ext }}(a)$. If one now uses, Equations (1), (40), (54) and (55), one finds then $e^{2 \Phi(a)}=\left[1-2 G M /\left(c^{2} a\right)-\Lambda_{\text {ext }} a^{2} / 3\right]$ and $[1-b(a) / a]=\left[1-2 G M /\left(c^{2} a\right)-\Lambda_{\text {ext }} a^{2} / 3\right]$ which can be simplified to

$$
\begin{aligned}
\Phi(a) & =\frac{1}{2} \ln \left(1-\frac{2 G M}{c^{2} a}-\frac{\Lambda_{\text {ext }}}{3} a^{2}\right), \\
b(a) & =\frac{2 G M}{c^{2}}+\frac{\Lambda_{\text {ext }}}{3} a^{3} .
\end{aligned}
$$

From Equation (57), one deduces that the mass of the wormhole is given by

$$
M=\frac{c^{2}}{2 G}\left(b(a)-\frac{\Lambda_{\mathrm{ext}}}{3} a^{3}\right) .
$$

(ii) Matching of the equations I: The surface pressure

We are going to consider the case where static interior observers measure zero tidal forces, i.e., $\Phi_{\text {int }}=$ constant, $\Phi_{\text {int }}^{\prime}=0$. This is, of course, a particular choice which simplifies the analysis. As we have seen, the metric is continuous through the surface $S$. However, their first and second derivatives might not be. Since the metric is static and spherically symmetric the only derivatives that one needs to worry about are radial. Now, second derivatives in the metric are related to the Einstein tensor $G_{\mu \nu}$, or since we are working with hat quantities, to $G_{\hat{\mu} \hat{\nu}}$. But $G_{\hat{\mu} \hat{\nu}}$ is proportional to the stress-energy tensor $T_{\hat{\mu} \hat{\nu}}$. Thus, something in the stress-energy tensor 
has to reflect this discontinuity. Indeed, at the boundary $S, T_{\hat{\mu} \hat{\nu}}$ is proportional to a Dirac $\delta$-function, and we can write $T_{\hat{\mu} \hat{\nu}}=t_{\hat{\mu} \hat{\nu}} \delta(\hat{r}-\hat{a})$, where $\hat{r}=\sqrt{g_{r r}} r$ means the proper distance through the thin shell. To find $t_{\hat{\mu} \hat{\nu}}$ one then uses $\int_{-}^{+} G_{\hat{\mu} \hat{\nu}} d \hat{r}=\left(8 \pi G / c^{4}\right) \int_{-}^{+} t_{\hat{\mu} \hat{\nu}} \delta(\hat{r}-\hat{a}) d \hat{r}$, where $\int_{-}^{+}$means an infinitesimal integral through the shell. Using the property of the $\delta$-function $\delta(f(x))=\left(1 /\left|f^{\prime}(x)\right|\right) \delta(x)$, and $\int_{-}^{+} g(x) \delta\left(x-x_{0}\right)=g\left(x_{0}\right)$, one finds

$$
t_{\hat{\mu} \hat{\nu}}=\frac{c^{4}}{8 \pi G} \int_{-}^{+} G_{\hat{\mu} \hat{\nu}} d \hat{r} .
$$

Since the shell is infinitesimally thin in the radial direction there is no radial pressure, thus we are left with a surface energy term $\Sigma$, and a surface tangential pressure $\mathcal{P}$.

First we calculate the surface energy density $\Sigma$. From Equation (6) we see that $G_{\hat{t} \hat{t}}$ only depends on first derivatives of the metric, so that when integrated through the shell it will give metric functions only, that by definition are continuous. Thus, since the integral gives the value of the metric on the exterior side $\left(b^{+}\right.$, say) minus the value of the metric on the interior side $\left(b^{-}\right)$, it gives zero, and one finds

$$
\Sigma=0 \text {. }
$$

Now we find the surface tangential pressure $\mathcal{P}$. From Equation (8) we see that $G_{\hat{\theta} \hat{\theta}}$ has an important term $\left(1-\frac{b}{r}\right) \Phi^{\prime \prime}$. The other terms depend at most on the first derivative and as before do no contribute to the integral. Thus, in this case Equation (59) gives $8 \pi G / c^{2} \mathcal{P}=\sqrt{1-b(a) / a} \Phi_{-}^{\prime+}$. Now, $\Phi_{-}^{\prime}=0$ by assumption, and $\Phi^{\prime+}=\left[G M /\left(c^{2} a^{2}\right)-\Lambda_{\text {ext }} a / 3\right] /(1-b(a) / a)$. Thus, $\mathcal{P}=\frac{c^{4}}{8 \pi G a} \frac{G M /\left(c^{2} a^{2}\right)-\Lambda_{\mathrm{ext}} a / 3}{\sqrt{1-b(a) / a}}$, or more explicitly,

$$
\mathcal{P}=\frac{c^{4}}{8 \pi G a} \frac{\frac{G M}{c^{2} a}-\frac{\Lambda_{\mathrm{ext}}}{3} a^{2}}{\sqrt{1-\frac{2 G M}{c^{2} a}-\frac{\Lambda_{\mathrm{ext}}}{3} a^{2}}} .
$$

$\mathcal{P}$ is always positive for the Schwarzschild and the Schwarzschild-anti de Sitter spacetime, i.e., $\Lambda_{\text {ext }} \leq 0$. The Schwarzschild-de Sitter spacetime, $\Lambda_{\text {ext }}>0$, has to be analyzed more carefully. In Figure 3 , we plot in a graph $9 \Lambda_{\text {ext }}\left(G M / c^{2}\right)^{2} \times 2 G M /\left(c^{2} a\right)$ the regions where $\mathcal{P}$ is negative, zero or positive. For high $\Lambda_{\text {ext }}\left(G M c^{-2}\right)^{2}$ (either $\Lambda_{\text {ext }}$ big or $M$ big) and at low 
$2 G M /\left(c^{2} a\right)$ (either $M$ small or $a$ big), one needs a surface tension to support the structure. In the other case one needs a surface pressure. This is expected in the sense that for a positive $\Lambda_{\text {ext }}$ one has an expanding external de Sitter spacetime. If $a$ is big (and so $2 G M /\left(c^{2} a\right)$ small), the wormhole boundary is participating somehow in the expansion, so one needs a tension to hold it. For small $a\left(\frac{2 G M}{c^{2} a}\right.$ big), the gravity wins over the expansion and so one needs a pressure to hold against collapse, a particular case being the Schwarzschild case $\Lambda_{\text {ext }}=0$.

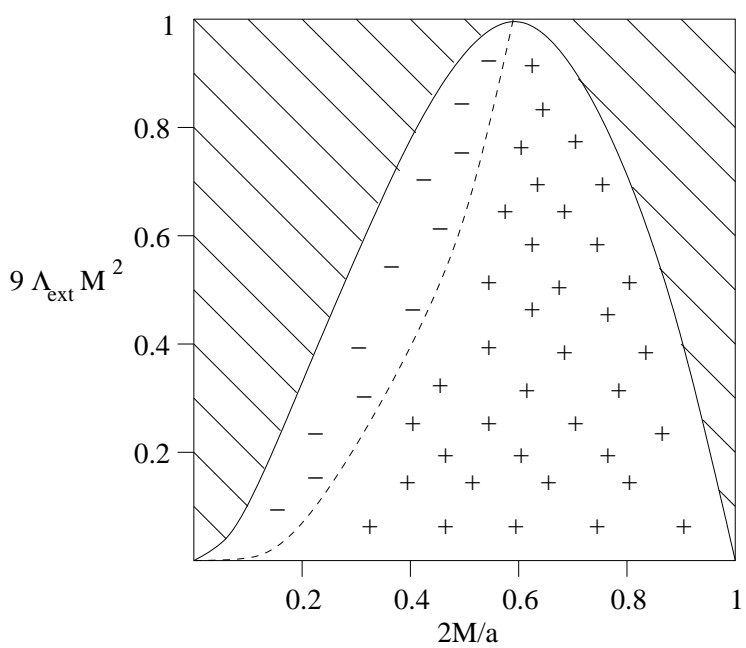

Figure 3 - The regions where $\mathcal{P}$ is negative and positive in a plot of $9 \Lambda_{\text {ext }}\left(G M / c^{2}\right)^{2}$ as a function of the inverse of the relative size of the wormhole, i.e., $2 G M / c^{2} a$ (in the figure we have used geometrical units $G=1=c$ ) are given. Inside the solid line is the region of solutions. To the left of the dashed line $\mathcal{P}$ is a negative pressure, i.e., a tension, to the right $\mathcal{P}$ is a positive quantity, i.e., is a pressure, see text for more details.

Now we detail the procedure to plot the graph. Define $\alpha=2 G M /\left(c^{2} a\right)$ and $\beta=9 \Lambda_{\text {ext }}\left(G M c^{-2}\right)^{2}$, so that $f=1-\frac{2 G M}{c^{2} a}-\frac{\Lambda_{\text {ext }}}{3} a^{2}$ can be written as $1-\alpha-\frac{4 \beta}{27 \alpha^{2}}$. The zero of $f$, defining the corresponding horizon for the set of parameters, gives $\beta$ as a function of $\alpha$. Varying $\alpha$ from 0 to 1 one gets the solid curve in Figure 3. The inside of this curve defines the region where $\mathcal{P}$ takes values (outside the curve there are no wormholes, on the top left hand part one mouth of the possible wormhole is causally disconnected from the other forever, and on the top right hand part the wormhole has been 
converted into a black hole ). We can check for the sign. To do this define $g=\frac{\alpha}{2}-\frac{4 \beta}{27 \alpha^{2}}$, see Equation (61). $g=0$ defines the curve $\beta=\frac{27}{8} \alpha^{3}$, which is plotted in the graph as a dashed line. To the left of this dashed curve, $\mathcal{P}$ is negative, and to the right it is positive.

One can have a term of comparison for the surface tangential pressure $\mathcal{P}$ at the thin shell. Assuming that the thin shell has a width of approximately $\Delta r$, one can consider a volumetric tangential pressure, orthogonal to the radial coordinate, given by

$$
\tilde{P}=\frac{\mathcal{P}}{\Delta r} .
$$

Taking into account Equation (35), we see that the tangential pressure at the mouth, with $\Phi_{\mathrm{int}}^{\prime}=0$, is given by $\bar{p}(a)=\left(c^{4} / 16 \pi G a^{3}\right)\left(b(a)-b^{\prime}(a) a\right)$. Estimates of $\tilde{P}$ may be given in terms of $p(a)$, by defining the following ratio

$$
\frac{\tilde{P}}{\bar{p}(a)}=2 \frac{a}{\Delta r} \frac{1}{\frac{b}{a}-b^{\prime}} \frac{\frac{G M}{c^{2} a}-\frac{\Lambda_{\mathrm{ext}}}{3} a^{2}}{\sqrt{1-\frac{2 G M}{c^{2}}-\frac{\Lambda_{\mathrm{ext}}}{3} a^{3}}} .
$$

It is also interesting to find the ratio to $p\left(r_{\mathrm{o}}\right)$, the maximum pressure, given by

$$
\frac{\tilde{P}}{p\left(r_{\mathrm{o}}\right)}=2 \frac{r_{\mathrm{o}}^{2}}{a \Delta r} \frac{1}{1-b^{\prime}\left(r_{\mathrm{o}}\right)} \frac{\frac{G M}{c^{2} a}-\frac{\Lambda_{\mathrm{ext}}}{3} a^{2}}{\sqrt{1-\frac{2 G M}{c^{2} a}-\frac{\Lambda_{\mathrm{ext}}}{3} a^{2}}} .
$$

One may find numerical estimates, considering various choices of the shape function, $b(r)$, which will be done while considering specific solutions of traversable wormholes.

(iii) Matching of the equations II: The radial pressure

To construct specific solutions of wormholes with generic cosmological constant, one needs to know how the radial tension behaves across the junction boundary, $S$. The analysis is simplified if we consider two general solutions of Equation (1), an interior solution and an exterior solution matched at a surface, $S$. The radial component of the Einstein equations, Equation (20), provides

$$
\begin{aligned}
& \frac{b_{\mathrm{int}}}{r^{3}}=\frac{8 \pi G}{c^{4}} \tau_{\mathrm{int}}(r)+\Lambda_{\mathrm{int}}+2\left(1-\frac{b_{\mathrm{int}}}{r}\right) \frac{\Phi_{\mathrm{int}}^{\prime}}{r}, \\
& \frac{b_{\mathrm{ext}}}{r^{3}}=\frac{8 \pi G}{c^{4}} \tau_{\mathrm{ext}}(r)+\Lambda_{\mathrm{ext}}+2\left(1-\frac{b_{\mathrm{ext}}}{r}\right) \frac{\Phi_{\mathrm{ext}}^{\prime}}{r} .
\end{aligned}
$$


Taking into account the continuity of the metric at the junction boundary one has obtained $\Phi_{\text {int }}(a)=\Phi_{\text {ext }}(a)$ and $b_{\text {int }}(a)=b_{\text {ext }}(a)$. For simplicity, we are considering $\Phi_{\text {int }}^{\prime}(a)=0$. Using again the following relation, $\Phi_{\text {ext }}^{\prime}(r)=$ $\left(\frac{G M}{c^{2} r^{2}}-\frac{\Lambda_{\mathrm{ext}}}{3} r\right) /\left(1-\frac{2 G M}{c^{2} r}-\frac{\Lambda_{\mathrm{ext}}}{3} r^{2}\right)$, and taking into account Equation (61), we verify that Equations (65)-(66), provide us with an equation which governs the behavior of the radial tension at the boundary, namely,

$$
\tau_{\mathrm{int}}(a)+\frac{c^{4}}{8 \pi G} \Lambda_{\mathrm{int}}=\tau_{\mathrm{ext}}(a)+\frac{c^{4}}{8 \pi G} \Lambda_{\mathrm{ext}}+\frac{2}{a} \mathcal{P} e^{\Phi(a)}
$$

where we have put $e^{\Phi(a)}=\sqrt{1-2 G M /\left(c^{2} a\right)-\Lambda_{\text {ext }} a^{2} / 3}$. Equation (67), although not new in its most generic form [2], is a beautiful equation that relates the radial tension at the surface with the tangential pressure of the thin shell. A particularly interesting case is when $\mathcal{P}=0$. In this situation $M=\Lambda_{\text {ext }} c^{2} a^{3} /(3 G)$, and $\Phi_{\text {ext }}^{\prime}(a)=0$. Since by our construction $\Phi_{\text {int }}^{\prime}(a)=0, \Phi^{\prime}$ is continuous across the surface and the solution is reduced to a boundary surface. From Equation (57) one finds that the shape function at the junction is given by $b(a)=\Lambda_{\text {ext }} a^{3}$. Thus, Equation (67) simplifies to, $\tau_{\text {int }}(a)+\frac{c^{4}}{8 \pi G} \Lambda_{\text {int }}=\tau_{\text {ext }}(a)+\frac{c^{4}}{8 \pi G} \Lambda_{\text {ext }}$. If one considers a matching of an interior solution of a wormhole with generic $\Lambda$, given by Equation (1), to an exterior Schwarzschild solution, with $\tau_{\text {ext }}=0$ and $\Lambda_{\text {ext }}=0$, we simply have the condition that $\tau_{\text {int }}(a)+\frac{c^{4}}{8 \pi G} \Lambda_{\text {int }}=0$ at the boundary surface. Matching an interior solution to an exterior Schwarzschild-de Sitter or Schwarzschild-anti de Sitter solution, with $\tau_{\text {ext }}=0$ and $\Lambda_{\text {ext }} \neq 0$, we have the following relation-

ship, $\tau_{\text {int }}(a)+\frac{c^{4}}{8 \pi G} \Lambda_{\text {int }}=\frac{c^{4}}{8 \pi G} \Lambda_{\text {ext }}$ at the boundary surface. These solutions will be analyzed in greater detail in the next sections.

\subsection{Spacetime diagrams}

We now draw the spacetime diagrams, i.e., the Carter-Penrose diagrams, corresponding to wormholes in spacetimes with $\Lambda_{\text {ext }}=0, \Lambda_{\text {ext }}>0$, and $\Lambda_{\text {ext }}<$ 0 . They are easy to sketch once one knows the corresponding diagrams for the solution with no wormhole, i.e, the Carter-Penrose diagrams for Minkowski spacetime, de Sitter spacetime and anti-de Sitter spacetime [8], respectively. Each point in the diagram represents a sphere. 


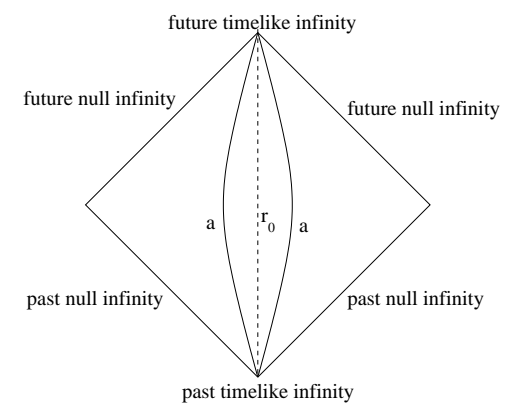

Figure 4 - The spacetime diagram for the wormhole with $\Lambda_{\text {ext }}=0$, i.e, a wormhole in an asymptotically Minkowski spacetime, represented by two copies of the Minkowski diagram joined at the throat.

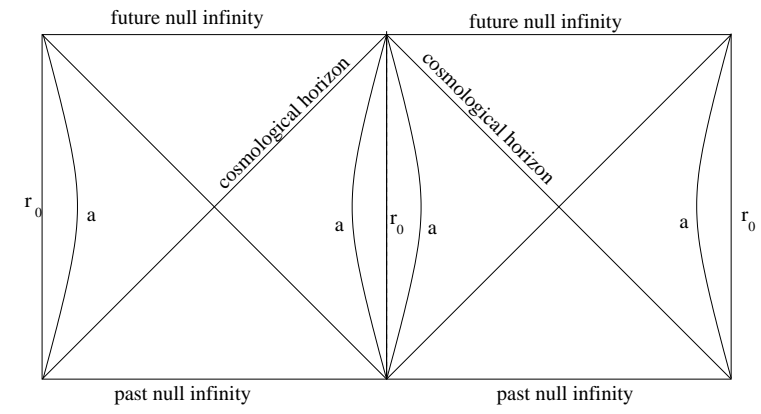

Figure 5 - The spacetime diagram for the wormhole with $\Lambda_{\text {ext }}>0$, i.e, a wormhole in an asymptotically de Sitter spacetime, with an infinite number of copies (only two are represented).

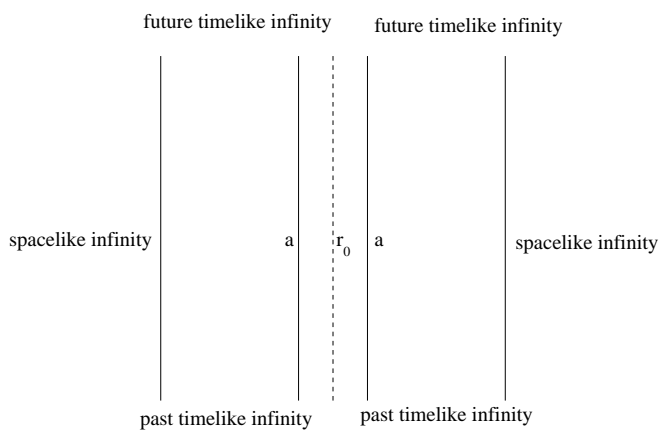

Figure 6 - The spacetime diagram for the wormhole with $\Lambda_{\text {ext }}<0$, i.e, a wormhole in an asymptotically anti-de Sitter spacetime, represented by two copies of the anti-de Sitter diagram joined at the throat. 
Since the wormhole creates an extra asymptotic region one has to duplicate the original diagram through the throat. In Figures 4, 5 and 6 the diagrams for a wormhole in an asymptotically flat spacetime $\Lambda_{\text {ext }}=0$, in an asymptotically de Sitter spacetime $\Lambda_{\text {ext }}>0$, and in an asymptotically anti-de Sitter

spacetime $\Lambda_{\text {ext }}<0$, respectively, are drawn. Note the duplication of the asymptotic regions.

\section{Specific construction of wormholes with generic $\Lambda$}

We will give some examples of traversable wormholes similar to those constructed in [1]. The difference from the wormholes in that work is that the wormholes here in general have an infinitesimal thin shell with a tangential pressure $\mathcal{P} \neq 0$, and the exterior spacetime has a cosmological constant. We discuss first the case $\Lambda_{\text {ext }}=0$, then $\Lambda_{\text {ext }}>0$, and finally we mention briefly the case $\Lambda_{\text {ext }}<0$. In all cases we put $\Phi^{\prime}=0$ in the interior region.

\subsection{Specific solutions of traversable wormholes with $\Lambda_{\text {ext }}=0$ (asymptotically flat wormholes)}

\subsubsection{Matching to an exterior Schwarzschild solution, with $\mathcal{P}=0$}

Here we consider a matching of an interior solution with an exterior Schwarzschild solution $\left(\tau_{\text {ext }}=0\right.$ and $\left.\Lambda_{\text {ext }}=0\right)$, and with the junction having zero tangential pressure, $\mathcal{P}=0$. From Equation (67) one has at the junction

$$
\tau_{\text {int }}(a)+\frac{c^{4}}{8 \pi G} \Lambda_{\text {int }}=0 .
$$

Then, from Equation (34) (with $\Phi^{\prime}=0$ ) one gets

$$
0=\frac{c^{4}}{8 \pi G} \frac{b(a)}{a^{3}} .
$$

Since $b \neq 0$, Equation (69) is only satisfied if $a \rightarrow \infty$. This is one of the cases considered by Morris and Thorne [1], in which the wormhole's material extends from the throat all the way to infinity. 


\subsubsection{Matching to an exterior Schwarzschild solution, with $\mathcal{P} \neq 0$}

Matching the interior solution to an exterior Schwarzschild solution $\left(\tau_{\text {ext }}=0\right.$ and $\Lambda_{\text {ext }}=0$ ) but considering $\mathcal{P} \neq 0$, provides some interesting results. The behavior of the radial tension at the junction is given by Equation (67) and taking into account Equation (57) one finds that the shape function at the junction simply reduces to $b(a)=2 G M / c^{2}$. We will next consider various choices for the shape function, $b(r)$, which will give different wormhole solutions.

(i) $b(r)=\left(r_{\mathrm{o}} r\right)^{1 / 2}$

Consider the following functions

$$
\begin{aligned}
\Phi(r) & =\Phi_{0}, \\
b(r) & =\left(r_{\mathrm{o}} r\right)^{1 / 2},
\end{aligned}
$$

where $r_{\mathrm{o}}$ is the throat radius as before. Using the Einstein equations, Equations (33)-(35) we have

$$
\begin{aligned}
& \bar{\rho}(r) \equiv \rho(r)+\frac{c^{2}}{8 \pi G} \Lambda_{\text {int }}=\frac{c^{2}}{8 \pi G} \frac{r_{\mathrm{o}}^{1 / 2}}{2 r^{5 / 2}}, \\
& \bar{\tau}(r) \equiv \tau(r)+\frac{c^{2}}{8 \pi G} \Lambda_{\text {int }}=\frac{c^{4}}{8 \pi G} \frac{r_{\mathrm{o}}^{1 / 2}}{r^{5 / 2}}, \\
& \bar{p}(r) \equiv p(r)+\frac{c^{2}}{8 \pi G} \Lambda_{\text {int }}=\frac{c^{4}}{8 \pi G} \frac{r_{\mathrm{o}}^{1 / 2}}{4 r^{5 / 2}} .
\end{aligned}
$$

The distribution of the material threading the wormhole is plotted in Figure 7. To find an estimate of the surface pressure at the thin shell, one has $\frac{\tilde{P}}{p\left(r_{\mathrm{o}}\right)}=\frac{4 r_{0}^{2} G M}{\Delta r c^{2} a^{2}}\left(1-\frac{2 G M}{c^{2} a}\right)^{-1 / 2}$ (see Equation (64)).

From $b(a)=2 G M / c^{2}$ and $b(a)=\left(r_{\mathrm{o}} a\right)^{1 / 2}$, one finds that the matching occurs at

$$
a=\frac{\left(2 G M / c^{2}\right)^{2}}{r_{\mathrm{o}}} .
$$

Now in order that the wormhole is not a black hole one has to impose $a>2 G M / c^{2}$. Then, from Equation (75) one finds $r_{\mathrm{o}}<2 G M / c^{2}$. From Equation (75), we also extract the mass of the wormhole, given by $M=$ $c^{2}\left(r_{\mathrm{o}} a\right)^{1 / 2} /(2 G)$. 

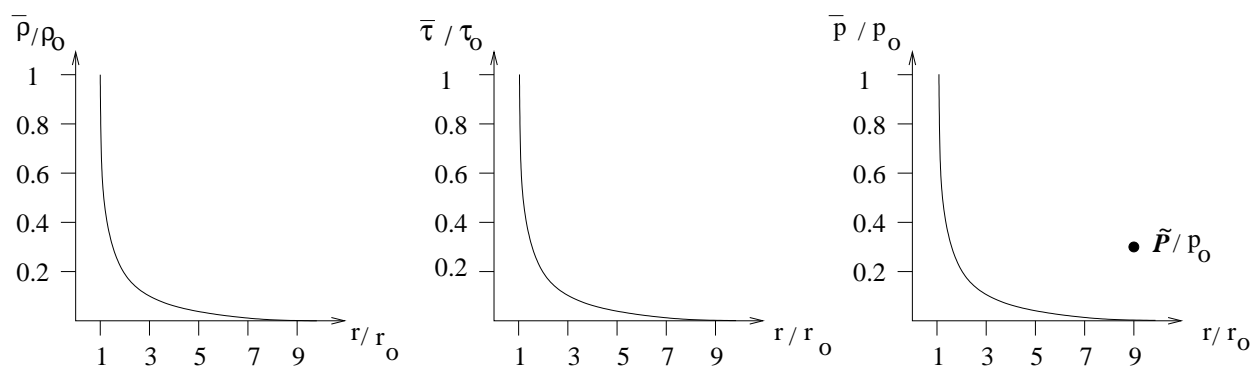

Figure 7 - Distribution of the material threading the wormhole for the case $b(r)=$ $\left(r_{\mathrm{o}} r\right)^{1 / 2}$. The mouth of the wormhole is at $a=9 r_{\mathrm{o}}$. The averaged surface pressure $\tilde{P}$ on the thin shell defined in the text is depicted qualitatively as $\tilde{P} / p\left(r_{\mathrm{o}}\right)$ in the $\bar{p} / p\left(r_{\mathrm{o}}\right)$ graph as a point.

The interior metric, $r_{\mathrm{o}} \leq r \leq a$, is determined recalling that $e^{2 \Phi(a)}=$ $\left[1-\sqrt{r_{\mathrm{o}} / a}\right]$. It is given by

$$
d s^{2}=-\left(1-\sqrt{\frac{r_{\mathrm{o}}}{a}}\right) c^{2} d t^{2}+\frac{d r^{2}}{\left(1-\sqrt{\frac{r_{\mathrm{o}}}{r}}\right)}+r^{2}\left(d \theta^{2}+\sin ^{2} \theta d \phi^{2}\right)
$$

The exterior metric, $a \leq r<\infty$, is given by

$$
d s^{2}=-\left(1-\frac{\sqrt{r_{\mathrm{o}} a}}{r}\right) c^{2} d t^{2}+\frac{d r^{2}}{\left(1-\frac{\sqrt{r_{\mathrm{o}} a}}{r}\right)}+r^{2}\left(d \theta^{2}+\sin ^{2} \theta d \phi^{2}\right) .
$$

The final metric of the whole spacetime is given by Equations (76)-(77), which are joined smoothly, as we have carefully worked out.

It is also interesting to briefly consider the traversability conditions that the absurdly advanced civilization might require to cross the wormhole from one mouth to the other and back (see the Appendix B for details). One finds that for an observer traversing the wormhole with a velocity $v=0.01 c$, the wormhole has a throat radius given by $r_{\mathrm{o}} \geq 500 \mathrm{~km}$. One can choose $r_{\mathrm{o}}=500 \mathrm{~km}$, and assume that the traversal time done by the spaceship is approximately one year. Then, one finds that the matter distribution extends from $r_{\mathrm{o}}$ to $a=4.74 \times 10^{13} \mathrm{~m} \approx 5 \times 10^{-3}$ light years, with $a$ being the size of the wormhole. It is supposed that the space stations are parked there. One also finds that the wormhole mass is $M=3.3 \times 10^{36} \mathrm{~kg}$, six orders of magnitude superior to the Sun's mass. One may also find an estimate for Equation (64), giving $\tilde{P} / p\left(r_{\mathrm{o}}\right) \approx 10^{-4}$. 
One may choose other parameters, for instance, so that the wormhole mass is of the same order of the Sun's mass. Considering a traversal with a velocity $v=5.4 \times 10^{3} \mathrm{~m} / \mathrm{s}$, we may choose that the wormhole throat is given by $r_{\mathrm{o}}=9 \times 10^{2} \mathrm{~m}$. If we consider an extremely fast trip, where the traversal time is given by $\Delta \tau_{\text {traveler }}=3.7 \mathrm{~s}$, the matter distribution extends from $r_{\mathrm{o}}$ to $a=10^{4} \mathrm{~m}$. In this case the mass of the wormhole is given by $M \approx 2 \times 10^{30} \mathrm{~kg}$, which is the Sun's mass. From Equation (64) an estimate to $\tilde{P}$ is $\tilde{P} / p\left(r_{\mathrm{o}}\right) \approx 5.7 \times 10^{3}$. We have an extremely large surface pressure. As the wormhole mass is decreased, one sees that a larger tangential surface pressure is needed to support the structure.

(ii) $b(r)=r_{\mathrm{o}}^{2} / r$

Consider now,

$$
\begin{aligned}
\Phi(r) & =\Phi_{0}, \\
b(r) & =r_{\mathrm{o}}^{2} / r .
\end{aligned}
$$

Using the Einstein equations, Equations (33)-(35), one has

$$
\begin{aligned}
& \bar{\rho}(r) \equiv \rho(r)+\frac{c^{2}}{8 \pi G} \Lambda_{\mathrm{int}}=-\frac{c^{2}}{8 \pi G} \frac{r_{\mathrm{o}}^{2}}{r^{4}}, \\
& \bar{\tau}(r) \equiv \tau(r)+\frac{c^{2}}{8 \pi G} \Lambda_{\mathrm{int}}=\frac{c^{4}}{8 \pi G} \frac{r_{\mathrm{o}}^{2}}{r^{4}}, \\
& \bar{p}(r) \equiv p(r)+\frac{c^{2}}{8 \pi G} \Lambda_{\mathrm{int}}=\frac{c^{4}}{8 \pi G} \frac{r_{\mathrm{o}}^{2}}{r^{4}} .
\end{aligned}
$$

The distribution of the material threading the wormhole is plotted in Figure 8. A qualitative estimate for $\mathcal{P} / p\left(r_{\mathrm{o}}\right)$, in this case given by $\frac{\tilde{P}}{p\left(r_{0}\right)}=$ $\frac{4 r_{0}^{2} G M}{\Delta r c^{2} a^{2}}\left(1-\frac{2 G M}{c^{2} a}\right)^{-1 / 2}$ (see Equation (64)) is also plotted.

From $b(a)=2 G M / c^{2}$ and $b(r)=r_{\mathrm{o}}^{2} / r$, the matching occurs at

$$
a=\frac{c^{2} r_{\mathrm{o}}^{2}}{2 G M} \text {. }
$$

Imposing $a>2 G M / c^{2}$, so that the wormhole does not correspond to a black hole solution, we have $r_{\mathrm{o}}>2 G M / c^{2}$ and the mass is given by $M=\frac{c^{2} r_{\mathrm{o}}^{2}}{2 G a}$. The interior metric, $r_{\mathrm{o}} \leq r \leq a$, with $e^{2 \Phi(a)}=\left[1-\left(r_{\mathrm{o}} / a\right)^{2}\right]$, is given by

$$
d s^{2}=-\left[1-\left(\frac{r_{\mathrm{o}}}{a}\right)^{2}\right] c^{2} d t^{2}+\frac{d r^{2}}{1-\frac{r_{0}^{2}}{r^{2}}}+r^{2}\left(d \theta^{2}+\sin ^{2} \theta d \phi^{2}\right) .
$$


The exterior metric, $a \leq r<\infty$, is given by

$$
d s^{2}=-\left(1-\frac{r_{\mathrm{o}}^{2}}{a r}\right) c^{2} d t^{2}+\frac{d r^{2}}{\left(1-\frac{r_{\mathrm{o}}^{2}}{a r}\right)}+r^{2}\left(d \theta^{2}+\sin ^{2} \theta d \phi^{2}\right)
$$

The respective final metric solution of the spacetime is given by Equations (84)-(85), joined smoothly at $a$.
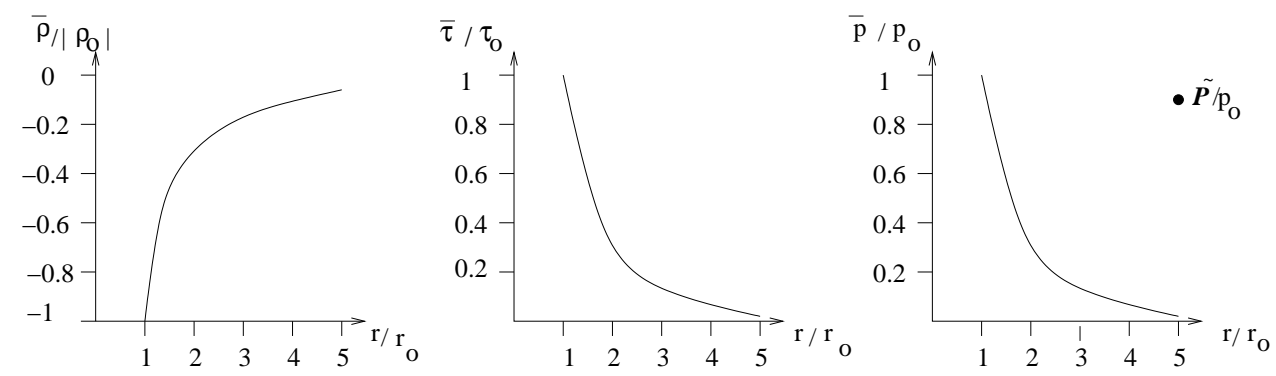

Figure 8 - Distribution of the material threading the wormhole for the case $b(r)=$ $r_{\mathrm{o}}^{2} / r$. The mouth of the wormhole is at $a=5 r_{\mathrm{o}} . \tilde{P} / p\left(r_{\mathrm{o}}\right)$ is depicted qualitatively in the $\bar{p} / p\left(r_{\mathrm{o}}\right)$ graph as a point.

A comment on the interior metric (84) is in order. In fact, this interior solution is the same as the one found by Ellis [18]. Indeed, considering the following coordinate transformations, $\bar{t}=\left[1-\left(r_{\mathrm{o}} / a\right)^{2}\right]^{1 / 2} t$ and $l= \pm\left(r^{2}-r_{\mathrm{o}}^{2}\right)^{1 / 2}$, one finds that the metric is reduced to $d s^{2}=-c^{2} d \bar{t}^{2}+$ $d l^{2}+\left(r_{\mathrm{o}}^{2}+l^{2}\right)\left(d \theta^{2}+\sin ^{2} \theta d \phi^{2}\right)$, where $l$ is the proper radial coordinate, ranging from $-\infty$ to $+\infty$. The properties are commented in [1] and [22]. Harris showed further that it is a solution of the Einstein equations with a stress-energy tensor of a peculiar massless scalar field [23].

With respect to the traversability conditions given in the Appendix B, considering that the traversal velocity is $v=0.01 c$, the wormhole throat is $r_{\mathrm{o}}=10^{6} \mathrm{~m}$. If the traversal time is one year, $\Delta \tau_{\text {traveler }}=3.16 \times 10^{7} \mathrm{~s}$, the junction is at $a=4.74 \times 10^{13} \mathrm{~m} \approx 5 \times 10^{-3}$ light years. The mass of the wormhole is $M \approx 1.4 \times 10^{25} \mathrm{~kg}$, approximately an order of magnitude superior to the Earth's mass. An estimate for Equation (64), is given by $\tilde{P} / p\left(r_{\mathrm{o}}\right) \approx 4.6 \times 10^{-16}$. 


\subsection{Specific solutions of traversable wormholes with $\Lambda_{\text {ext }}>0$ (asymptotically de Sitter wormholes)}

\subsubsection{Matching to an exterior Schwarzschild-de Sitter solution, with $\mathcal{P}=0$}

In this section we will be interested in a matching of an interior solution with an exterior Schwarzschild-de Sitter solution, $\tau_{\text {ext }}=0$ and $\Lambda_{\text {ext }}>0$, at a boundary surface, $\mathcal{P}=0$. We verify from Equation (67) that the following condition holds

$$
\tau_{\text {int }}(a)+\frac{c^{4}}{8 \pi G} \Lambda_{\text {int }}=\frac{c^{4}}{8 \pi G} \Lambda_{\text {ext }},
$$

at the surface boundary. Considering Equation (34), we have

$$
b(a)=\Lambda_{\text {ext }} a^{3} .
$$

Substituting this value in Equation (58), one obtains the mass of the wormhole, given by,

$$
M=\frac{c^{2}}{3 G} \Lambda_{\text {ext }} a^{3} .
$$

We shall next consider identical shape functions as in the above section.

(i) $b(r)=\left(r_{\mathrm{o}} r\right)^{1 / 2}$

Consider the following functions

$$
\begin{aligned}
\Phi(r) & =\Phi_{0}, \\
b(r) & =\left(r_{\mathrm{o}} r\right)^{1 / 2} .
\end{aligned}
$$

From $b(a)=\Lambda_{\mathrm{ext}} a^{3}$ and $b(a)=\left(r_{\mathrm{o}} a\right)^{1 / 2}$, one sees that the matching occurs at

$$
a \equiv \frac{r_{\mathrm{o}}^{1 / 5}}{\Lambda_{\mathrm{ext}}^{2 / 5}} .
$$

The mass can then be expressed as $M=c^{2}\left(r_{\mathrm{o}} a\right)^{1 / 2} /(3 G)$. It can be shown that the interior solution, $r_{\mathrm{o}} \leq r \leq a$, is identical to Equation (76), i.e.,

$$
d s^{2}=-\left(1-\sqrt{\frac{r_{\mathrm{o}}}{a}}\right) c^{2} d t^{2}+\frac{d r^{2}}{\left(1-\sqrt{\frac{r_{\mathrm{o}}}{r}}\right)}+r^{2}\left(d \theta^{2}+\sin ^{2} \theta d \phi^{2}\right) .
$$


The exterior solution, $a \leq r<\infty$, is given by the following metric

$$
\begin{gathered}
d s^{2}=-\left(1-\frac{2\left(r_{\mathrm{o}} a\right)^{1 / 2}}{3 r}-\frac{r_{\mathrm{o}}^{1 / 2} r^{2}}{3 a^{5 / 2}}\right) c^{2} d t^{2}+\frac{d r^{2}}{\left(1-\frac{2\left(r_{\mathrm{o}} a\right)^{1 / 2}}{3 r}-\frac{r_{\mathrm{o}}^{1 / 2} r^{2}}{3 a^{5 / 2}}\right)} \\
+r^{2}\left(d \theta^{2}+\sin ^{2} \theta d \phi^{2}\right) .
\end{gathered}
$$

The spacetime of the final solution is given by the metrics, Equations (92)(93), which have been smoothly joined at $a$.

The additional parameter now is the cosmological constant, $\Lambda_{\text {ext }}$, given by $\Lambda_{\text {ext }}=\left(r_{\mathrm{o}} / a^{5}\right)^{1 / 2}$. For instance, consider a traversal velocity $v=0.01 c$, so that $r_{\mathrm{o}}=5 \times 10^{5} \mathrm{~m}$. If the observer traverses through the wormhole comfortably during a year, $\Delta \tau_{\text {traveler }} \approx 3.16 \times 10^{7} \mathrm{~s}$, and $a=4.74 \times 10^{13} \mathrm{~m}$. The mass of the wormhole is $M \approx 2.2 \times 10^{36} \mathrm{~kg}$ and the cosmological constant has the value $\Lambda_{\text {ext }}=4.6 \times 10^{-32} \mathrm{~m}^{-2}$. The cosmological event horizon is then situated at $r_{c}=8.1 \times 10^{15} \mathrm{~m} \approx 200 a$.

(ii) $b(r)=r_{\mathrm{o}}^{2} / r$

Consider now the functions

$$
\begin{aligned}
\Phi(r) & =\Phi_{0}, \\
b(r) & =r_{\mathrm{o}}^{2} / r .
\end{aligned}
$$

From $b(a)=\Lambda_{\text {ext }} a^{3}$ and $b(a)=r_{\mathrm{o}}^{2} / a$ one sees that the matching occurs at $a=r_{\mathrm{o}}^{1 / 2} / \Lambda_{\text {ext }}^{1 / 4}$. The mass can be written as $M=c^{2} r_{\mathrm{o}}^{2} /(3 G a)$. The exterior cosmological constant is then $\Lambda_{\text {ext }}=\left(r_{\mathrm{o}} / a^{2}\right)^{2}$.

Considering that the traversal velocity is $v=0.01 c$, the throat radius is $r_{\mathrm{o}}=10^{6} \mathrm{~m}$. If the traversal time is done in one year the matching occurs at $a=4.74 \times 10^{13} \mathrm{~m}$. The mass is then given by $M=9.5 \times 10^{24} \mathrm{~kg}$, the same order of magnitude as the Earth's mass, and the cosmological constant is $\Lambda_{\text {ext }}=1.98 \times 10^{-43} \mathrm{~m}^{-2}$. The cosmological horizon is situated at $r_{c}=$ $3.9 \times 10^{21} \mathrm{~m} \approx 10^{8} a$.

Another example is provided by a traveler with velocity is $v=0.0001 \mathrm{c}$. The throat radius is then $r_{\mathrm{o}}=10^{4} \mathrm{~m}$. Assuming that the traversal time is done in $\Delta \tau_{\text {traveler }}=6.3 \times 10^{3}$ years, one has $a \approx 3 \times 10^{15} \mathrm{~m} \approx 0.32$ light years. The mass is given by $M=1.5 \times 10^{19} \mathrm{~kg}$, the mass of an asteroid, and the cosmological constant has a value of $\Lambda_{\text {ext }}=10^{-54} \mathrm{~m}^{-2}$, approximately the present value. 


\subsubsection{Matching to an exterior Schwarzschild-de Sitter solution, with $\mathcal{P} \neq 0$}

One can also match the interior solution with an exterior Schwarzschild-de Sitter solution $\left(\tau_{\text {ext }}=0\right.$ and $\left.\Lambda_{\text {ext }}>0\right)$ in the presence of a thin shell, $\mathcal{P} \neq 0$. From Equation (67), we have the behavior of the radial tension at the thin shell, given by

$$
\tau_{\text {int }}(a)+\frac{c^{4}}{8 \pi G} \Lambda_{\text {int }}=\frac{c^{4}}{8 \pi G} \Lambda_{\text {ext }}+\frac{2}{a} \mathcal{P} e^{\Phi(a)} .
$$

The shape function at the junction is given by Equation (57). From Equation (58), one verifies that the mass of the wormhole is zero when $b(a)=\Lambda_{\text {ext }} a^{3} / 3$, is positive when $b(a)>\Lambda_{\text {ext }} a^{3} / 3$, and is negative when $b(a)<\Lambda_{\text {ext }} a^{3} / 3$. One can perform a similar analysis as done for the previous examples.

\subsection{Specific solutions of traversable wormholes with $\Lambda_{\text {ext }}<0$ (asymptotically anti-de Sitter wormholes)}

\subsubsection{Matching to an exterior Schwarzschild-anti de Sitter solu- tion, with $\mathcal{P}=0$}

From Equation (67), matching an interior solution with an exterior given by the Schwarzschild-anti de Sitter solution $\left(\tau_{\text {ext }}=0\right.$ and $\left.\Lambda_{\text {ext }}<0\right)$, at a boundary surface $\mathcal{P}=0$, yields,

$$
\tau_{\text {int }}(a)+\frac{c^{4}}{8 \pi G} \Lambda_{\text {int }}=-\frac{c^{4}}{8 \pi G}\left|\Lambda_{\text {ext }}\right|,
$$

at the surface boundary. Considering Equation (34), we have

$$
b(a)=-\left|\Lambda_{\text {ext }}\right| a^{3} .
$$

From Equation (26), we concluded that the shape function has to be positive to guarantee that the factor $\sqrt{r / b-1}$ be real. Therefore, for the anti-de Sitter exterior, i.e., $\Lambda_{\text {ext }}<0$, with $\mathcal{P}=0$ there is no solution. This problem may be overcome by considering a matching to an exterior anti-de Sitter solution with a thin shell, i.e., $\mathcal{P} \neq 0$. 


\subsubsection{Matching to an exterior Schwarzschild-anti de Sitter solu- tion, with $\mathcal{P} \neq 0$}

From Equation (57), one finds that $b(a)$ is positive if

$$
\frac{2 G M}{c^{2}} \geq \frac{\left|\Lambda_{\text {ext }}\right|}{3} a^{3} .
$$

Then one can construct easily wormholes in anti-de Sitter spacetime, and again perform a similar analysis as done for the previous examples.

\section{Conclusions}

We have considered Morris-Thorne wormholes, i.e., static and spherically symmetric traversable wormholes, in the presence of a non-vanishing cosmological constant. Matching the interior solution with a vacuum exterior solution, we have deduced an equation for the tangential surface pressure, and another one which governs the behavior of the radial tension at the boundary.

Specific solutions with various choices of the shape function were presented. Through the traversability conditions, we have obtained estimates for the matching boundary, $a$, the mass of the wormhole $M$, and the tangential surface pressure $\mathcal{P}$, by imposing values for the traversal velocity and the traversal time.

Acknowledgements JPSL thanks Joseph Katz and Donald Lynden-Bell for teaching many years ago how to do junctions in an easy way, thanks Madalena Pizarro for alerting that a civilization that constructs a wormhole is an absurdly advanced civilization, rather than arbitrarily advanced, and thanks Observatório Nacional do Rio de Janeiro for hospitality. FSNL thanks many conversations with Paulo Crawford do Nascimento. The present address of SQO is Instituto de Física, Universidade Federal do Rio de Janeiro,

CEP 21945-970, Rio de Janeiro. This work was partially funded through project PESO/PRO/2000/4014 by Fundação para a Ciência e Tecnologia (FCT) - Portugal. 


\section{A Solutions of $\left(1-\frac{2 G M}{c^{2} r}-\frac{\Lambda_{\mathrm{ext}}}{3} r^{2}\right)=0$}

The roots of the cubic equation

$$
x^{3}+a x+b=0,
$$

can be found. Indeed, defining

$$
\begin{aligned}
A & =\sqrt[3]{-\frac{b}{2}+\sqrt{\frac{b^{2}}{4}+\frac{a^{3}}{27}}}, \\
B & =\sqrt[3]{-\frac{b}{2}-\sqrt{\frac{b^{2}}{4}+\frac{a^{3}}{27}}},
\end{aligned}
$$

the solutions are given by

$$
\begin{aligned}
& x_{1}=A+B \\
& x_{2}=-\frac{A+B}{2}+\frac{A-B}{2} \sqrt{-3}, \\
& x_{3}=-\frac{A+B}{2}-\frac{A-B}{2} \sqrt{-3} .
\end{aligned}
$$

(i) The Schwarzschild spacetime, $\Lambda_{\mathrm{ext}}=0$ :

This case is trivial and is analyzed directly in the text.

(ii) The Schwarzschild-de Sitter spacetime, $\Lambda_{\mathrm{ext}}>0$ :

The equation $f(r)=\left(1-\frac{2 G M}{c^{2} r}-\frac{\Lambda_{\mathrm{ext}}}{3} r^{2}\right)=0$ may be recast into the form

$$
x^{3}-\sigma x+\sigma=0,
$$

with $x=r /\left(2 G M c^{-2}\right)$ and $\sigma=3 c^{4} /\left(4 \Lambda_{\text {ext }} G^{2} M^{2}\right)$. Thus comparing (106) with (100) one finds

$$
\begin{aligned}
& A=\sqrt[3]{-\frac{\sigma}{2}+\sqrt{\frac{\sigma^{2}}{4}-\frac{\sigma^{3}}{27}}} \\
& B=\sqrt[3]{-\frac{\sigma}{2}-\sqrt{\frac{\sigma^{2}}{4}-\frac{\sigma^{3}}{27}}}
\end{aligned}
$$


To find which two of the three solutions one must pick up, we consider the case $\Lambda_{\text {ext }}\left(G M / c^{2}\right)^{2} \ll 1$, i.e., $\sigma \gg 1$. The square root $\sqrt{\frac{\sigma^{2}}{4}-\frac{\sigma^{3}}{27}}$ may be expanded as $i \frac{\sigma^{3 / 2}}{\sqrt{27}}\left(1-\frac{27}{8 \sigma}\right)$. Thus Equations (107)-(108) yield the following approximation

$$
\begin{aligned}
A & =\sqrt[3]{i} \frac{\sigma^{1 / 2}}{\sqrt{3}}\left(1+i \frac{\sqrt{27}}{6} \frac{1}{\sigma^{1 / 2}}\right) \\
B & =\sqrt[3]{-i} \frac{\sigma^{1 / 2}}{\sqrt{3}}\left(1-i \frac{\sqrt{27}}{6} \frac{1}{\sigma^{1 / 2}}\right)
\end{aligned}
$$

Defining $\alpha=\frac{\sigma^{1 / 2}}{\sqrt{3}}$ and $\beta=\frac{\sqrt{27}}{6} \frac{1}{\sigma^{1 / 2}}$, and considering $\sqrt[3]{i}=e^{i \frac{\pi}{6}}=\frac{\sqrt{3}}{2}+\frac{i}{2}$, $\sqrt[3]{-i}=e^{-i \frac{\pi}{6}}=\frac{\sqrt{3}}{2}-\frac{i}{2}$, Equations (109)-(110) take the form

$$
\begin{aligned}
& A=\alpha\left[\left(\frac{\sqrt{3}}{2}-\frac{\beta}{2}\right)+i\left(\frac{1}{2}+\frac{\sqrt{3}}{2} \beta\right)\right], \\
& B=\alpha\left[\left(\frac{\sqrt{3}}{2}-\frac{\beta}{2}\right)-i\left(\frac{1}{2}+\frac{\sqrt{3}}{2} \beta\right)\right] .
\end{aligned}
$$

We are only interested in the positive solutions. Thus, substituting Equations (111)-(112) into Equations (103)-(105) and taking into account $x=$ $r /\left(2 G M c^{-2}\right)$, we have that $x_{3}$ and $x_{1}$ give, respectively

$$
\begin{aligned}
& r_{b}=\frac{2 G M}{c^{2}}\left[1+\frac{4}{3} \Lambda_{\mathrm{ext}}\left(\frac{G M}{c^{2}}\right)^{2}\right], \\
& r_{c}=\sqrt{\frac{3}{\Lambda_{\mathrm{ext}}}}\left(1-\frac{G M}{c^{2}} \sqrt{\frac{\Lambda_{\mathrm{ext}}}{3}}\right) .
\end{aligned}
$$

We see that $r_{b}$ and $r_{c}$ correspond to the vacuum black hole horizon and to the cosmological event horizon, respectively. In Equation (113) to find the numerical factor of the first order term $\Lambda_{\text {ext }}\left(\frac{G M}{c^{2}}\right)^{2}$ it is easier to linearize the solution by writing $x=1+\epsilon$, for small $\epsilon$, and then with the help of (106) one finds $\epsilon$.

(iii) The Schwarzschild-anti de Sitter spacetime, $\Lambda_{\mathrm{ext}}<0$ :

In the Schwarzschild-anti de Sitter spacetime, one finds that the equation $f(r)=\left(1-\frac{2 G M}{c^{2} r}+\frac{\left|\Lambda_{\text {ext }}\right|}{3} r^{2}\right)=0$ may be recast into the form

$$
x^{3}+\sigma x-\sigma=0,
$$


with $x=r /\left(2 G M c^{-2}\right)$ and $\sigma=3 c^{4} /\left(4\left|\Lambda_{\text {ext }}\right| G^{2} M^{2}\right),\left|\Lambda_{\text {ext }}\right| \equiv-\Lambda_{\text {ext }}$. Thus comparing (115) with (100) one finds

$$
\begin{aligned}
& A=\sqrt[3]{\frac{\sigma}{2}+\sqrt{\frac{\sigma^{2}}{4}+\frac{\sigma^{3}}{27}}}, \\
& B=\sqrt[3]{\frac{\sigma}{2}-\sqrt{\frac{\sigma^{2}}{4}+\frac{\sigma^{3}}{27}}} .
\end{aligned}
$$

The only real solution is $x_{1}=A+B$. Indeed, for $\left|\Lambda_{\text {ext }}\right|\left(G M / c^{2}\right)^{2} \ll 1$, i.e., $\sigma \gg 1$ one finds

$$
r_{b}=\frac{2 G M}{c^{2}}\left[1-\frac{4}{3}\left|\Lambda_{\mathrm{ext}}\right|\left(\frac{G M}{c^{2}}\right)^{2}\right],
$$

which gives the black hole horizon. In Equation (118) to find the numerical factor of the first order term $\Lambda_{\text {ext }}\left(\frac{G M}{c^{2}}\right)^{2}$ it is easier to linearize the solution by writing $x=1+\epsilon$, for small $\epsilon$, and then with the help of (115) one finds $\epsilon$.

\section{B Traversability conditions}

We will be interested in specific solutions for traversable wormholes and assume that a traveler of an absurdly advanced civilization, with human traits, begins the trip in a space station in the lower universe, at proper distance $l=-l_{1}$, and ends up in the upper universe, at $l=l_{2}$. We shall, for self-containment and self-consistency, briefly describe the traversability conditions given in [1].

The cosmological constant does not enter the analysis directly. Indeed, although the interior cosmological constant, $\Lambda_{\text {int }}$, can be incorporated into the effective quantities, for $\Lambda_{\text {ext }} \neq 0$ the external parameters such as the mass of the wormhole change. Thus, the cosmological constant enters the traversability conditions indirectly. Ford and Roman [12] with their quantum inequalities have imposed severe restrictions on the $T_{\mu \nu}$ obeyed by matter, and in particular found that the parameters of the Morris-Thorne-Yurtsever [63] wormhole obeyed these inequalities. However, we now know that the energy conditions can be even classically violated [14], and thus the Ford-Roman inequalities have somehow lost their strength in this context. Therefore, our 
parameters are not chosen in order to satisfy the quantum inequalities, but rather follow the spirit of the Morris and Thorne work [1] where attention is paid to the traversability conditions of a human being.

\section{(i) Tidal acceleration felt by a traveler}

The tidal accelerations between two parts of the traveler's body, separated by, say, 2 meters, should not exceed the gravitational acceleration at Earth's surface $g_{\text {Earth }}$, (with $g_{\text {Earth }} \approx 10 \mathrm{~m} / \mathrm{s}^{2}$ ). From [1] and using our simplifying assumption $\Phi^{\prime}=0$, one obtains the following inequality

$$
\left|\frac{\gamma^{2}}{2 r^{2}}\left(\frac{v}{c}\right)^{2}\left(b^{\prime}-\frac{b}{r}\right)\right| \leq \frac{g_{\text {Earth }}}{2 c^{2} 1 \mathrm{~m}} \approx \frac{1}{10^{16} \mathrm{~m}^{2}},
$$

with $\gamma=\left(1-v^{2} / c^{2}\right)^{-1 / 2}$, and $v$ being the traveler's velocity. This inequality refers to tangential tidal accelerations. Radial tidal acceleration are zero for $\Phi^{\prime}=0$. From Equation (119) one sees that stationary observers with $v=0$ measure null tidal forces.

\section{(ii) Acceleration felt by a traveler}

Another condition that needs to be respected is that the local gravitational acceleration, $|\vec{a}|$ at the space stations should not surpass the terrestrial gravitational acceleration, $g_{\text {Earth }}$. The condition imposed in [1] $\left(\right.$ for $\left.\Phi^{\prime}=0\right)$ is

$$
|\vec{a}|=\left|\left(1-\frac{b}{r}\right)^{1 / 2} \gamma^{\prime} c^{2}\right| \leq g_{\text {Earth }}
$$

For $v=$ constant travelers, one has $|\vec{a}|=0$, of course!

(iii) Total time in a traversal

The trip should take a relatively short time, for instance one year, as measured by the traveler and for observers that stay at rest at the space stations, $l=-l_{1}$ and $l=l_{2}$, i.e.,

$$
\begin{aligned}
\Delta \tau_{\text {traveler }} & =\int_{-l_{1}}^{+l_{2}} \frac{d l}{v \gamma} \leq 1 \text { year } \\
\Delta t_{\text {space station }} & =\int_{-l_{1}}^{+l_{2}} \frac{d l}{v e^{\Phi}} \leq 1 \text { year }
\end{aligned}
$$


respectively.

Having set these conditions for general wormholes, we will now study two particular cases.

\section{(1) The wormhole with shape function $b(r)=\left(r_{\mathrm{o}} r\right)^{1 / 2}$}

\section{(i) Tidal acceleration felt by a traveler}

In the interior region, $r_{\mathrm{o}}<r<a$, the tidal acceleration as measured by a traveler moving radially through the wormhole is given by Equation (119). For non-relativistic velocities, $v \ll c$, we have $\gamma \approx 1$, and substituting the expression of $b(r)$, i.e., $b(r)=\left(r_{\mathrm{o}} r\right)^{1 / 2}$, in Equation (119), one can impose a velocity for the traveler traversing through the wormhole, such that the tidal accelerations felt are inferior to the terrestrial gravitational acceleration, $g_{\text {Earth }}$. With these conditions, one obtains a restriction for the velocity at the throat, where the acceleration is severest, given by

$$
\left(\frac{v}{c}\right) \leq \frac{2 r_{\mathrm{o}}}{10^{8} \mathrm{~m}}
$$

If the observer traverses the wormhole with a non-relativistic velocity $v \ll c$, the accelerations at the beginning and end of the trip are negligible. If we consider that the velocity is $v \approx 0.01 c$, one can find estimates for the dimensions of the wormhole. The wormhole throat obeys $r_{\mathrm{o}} \geq 500 \mathrm{~km}$. For definiteness consider the choice $r_{\mathrm{o}}=500 \mathrm{~km}$. Taking into account Equation (75) for asymptotically flat spacetimes, the region of matter distribution will extend to

$$
a=\frac{\left(2 G M / c^{2}\right)^{2}}{(500 \mathrm{~km})}
$$

Then, by choosing $a$, we find the value of the wormhole mass. We now take the steps to choose $a$. Similar procedures follow for $\Lambda_{\text {ext }} \neq 0$, see main text.

(ii) Acceleration felt by a traveler

Another condition that needs to be respected is that the local gravitational acceleration $|\vec{a}|$, at the space stations should not surpass the terrestrial gravitational acceleration, $g_{\text {Earth }}$. Considering non-relativistic velocities, $v \ll c$, and $v \approx$ constant, one has $|\vec{a}| \approx 0$, so that $\gamma \approx 1$. Condition (120) is immediately satisfied, the traveler feels a zero gravitational acceleration. 
(iii) Total time in a traversal

The expressions for the total times in a traversal of the wormhole, measured by the traveler and observers at rest at the station, are given by Equations (121)-(122). $l=-l_{1}$ and $l=l_{2}$ are the positions of the space stations. For low velocities, $v \ll c$, we have $\gamma \approx 1$, and with $\Phi=\Phi_{0}$, Equations (121)-(122) reduce to

$$
\Delta \tau_{\text {traveler }} \approx \frac{2 l}{v}=e^{\Phi_{0}} \Delta t_{\text {space station }}
$$

Suppose that the space stations are placed in the neighborhood of the mouth, at $a$, in the exterior side. It is convenient to place the space stations at large enough radii, i.e., $a \gg r_{\mathrm{o}}$, so that the factor $1-b(r) / r \approx 1$. Thus, one also has that $e^{2 \Phi(a)}=1-\left(r_{\mathrm{o}} / a\right)^{1 / 2} \approx 1$, so that $\Delta \tau_{\text {traveler }} \approx \Delta t_{\text {space station }} \approx 2 a / v$. Assume that the traversal time should be less than a year, i.e. $\Delta \tau_{\text {traveler }} \leq$ 1 year $\left(1\right.$ year $\left.=3.16 \times 10^{7} \mathrm{~s}\right)$. Therefore, from $2 a / v \approx 1$ year, one extracts a value for $a$, namely $a \approx 4.74 \times 10^{13} \mathrm{~m}$.

\section{(2) The wormhole with shape function $b(r)=r_{\mathrm{o}}^{2} / r$}

Applying a similar analysis as before, Equation (119), with $v \ll c$ and $\gamma \approx 1$, and considering the form function given by $b(r)=r_{\mathrm{o}}^{2} / r$, we have

$$
\left(\frac{v}{c}\right) \leq\left(\frac{r^{2}}{r_{\mathrm{o}}}\right) \frac{1}{10^{8} \mathrm{~m}}
$$

Imposing a traversal velocity $v=0.01 c$ at the throat, $r=r_{\mathrm{o}}$, one finds $r_{\mathrm{o}} \approx 10^{6} \mathrm{~m}$. For $\Phi=\Phi_{0}$ the local gravitational accelerations at the space stations are zero. The traversal time, with $\gamma \approx 1$ is given by

$$
\Delta \tau_{\text {traveler }} \approx \frac{2 l}{v}=e^{\Phi_{0}} \Delta t_{\text {space station }}
$$

By a similar analysis as above, we place the space stations at $a \gg r_{\mathrm{o}}$, implying that $e^{2 \Phi(a)}=1-\left(r_{\mathrm{o}} / a\right)^{2} \approx 1$, so that $\Delta \tau_{\text {traveler }} \approx \Delta t_{\text {spacestation. }}$. Considering that the traversal time is approximately one year, $\Delta \tau_{\text {traveler }}=3.16 \times 10^{7} \mathrm{~s}$, and taking into account that the traversal velocity is $v=0.01 c$, the junction surface is at $a=4.74 \times 10^{13} \mathrm{~m}$. 


\section{References}

[1] M. S. Morris and K. S. Thorne, "Wormholes in spacetime and their use for interstellar travel: A tool for teaching General Relativity", Am. J. Phys. 56, 395 (1988).

[2] M. Visser, Lorentzian Wormholes: From Einstein to Hawking (American Institute of Physics, New York, 1995).

[3] J. A. Wheeler, Geometrodynamics, (Academic Press, New York, 1962).

[4] S. W. Hawking, "Wormholes in spacetime", Phys. Rev. D 37, 904 (1988).

[5] R. P. Geroch, "Topology in General Relativity", J. Math. Phys. 8, 782 (1967).

[6] B. K. Harrison, K. S. Thorne, M. Wakano and J. A. Wheeler, Gravitational Theory and Gravitational Collapse, (University of Chicago Press, Chicago, 1965).

[7] Ya. B. Zel'dovich and I. D. Novikov, Relativistic Astrophysics, Vol. I: Stars and Relativity, (University of Chicago Press, Chicago, 1971).

[8] S. W. Hawking and G.F.R. Ellis, The Large Scale Structure of Spacetime, (Cambridge University Press, Cambridge 1973).

[9] G. Klinkhammer, "Averaged energy conditions for free scalar fields in flat spacetime", Phys. Rev. D 43, 2542 (1991).

[10] T. A. Roman, "Quantum stress-energy tensors and the weak energy condition", Phys. Rev. D 33, 3526 (1986).

[11] L. H. Ford and T. A. Roman, "Averaged energy conditions and quantum inequalities", Phys. Rev. D 51, 4277 (1995).

[12] L. H. Ford and T. A. Roman, "Quantum field theory constrains traversable wormhole geometries", Phys. Rev. D 53, 5496 (1996).

[13] L. H. Ford and T. A. Roman, "The quantum interest conjecture", Phys. Rev. D 60, 104018 (1999). 
[14] C. Barceló and M. Visser, "Scalar fields, energy conditions and traversable wormholes", Class. Quantum Grav. 17, 3843 (2000).

[15] B. McInnes, "The Covariant Entropy Bound, Brane Cosmology, and the Null Energy Condition, JHEP 0212, 053 (2002)

[16] D. Hochberg, A. Popov and S. Sushkov, "Self-consistent wormhole solutions of semiclassical gravity", Phys. Rev. Lett. 78, 2050 (1997).

[17] C. Barceló and M. Visser, "Traversable wormholes from massless conformally coupled scalar fields", Phys. Lett. B466, 127 (1999).

[18] H. G. Ellis, "Ether flow through a drainhole: A particle model in general relativity", J. Math. Phys. 14, 104 (1973).

[19] K. A. Bronnikov, "Scalar-tensor theory and scalar charge", Acta Phys. Pol. B 4, 251 (1973).

[20] T. Kodama, "General-relativistic nonlinear field: A kink solution in a generalized geometry", Phys. Rev. D 18, 3529 (1978).

[21] G. Clément, "Einstein-Yang-Mills-Higgs solitons", Gen. Rel. Grav. 13, 763 (1981).

[22] G. Clément, "The Ellis geometry", Am. J. Phys. 57, 967 (1989).

[23] E. G. Harris, "Wormhole connecting two Reissner-Nordström universes", Am. J. Phys. 61, 1140 (1993).

[24] M. Visser, "Traversable wormholes: Some simple examples", Phys. Rev. D 39, 3182 (1989).

[25] M. Visser, "Traversable wormholes: The Roman ring", Phys. Rev. D 55, 5212 (1997).

[26] M. Visser and D. Hochberg, "Generic wormhole throats", gr-qc/971001.

[27] N. Dadhich, S. Kar, S. Mukherjee and M. Visser, " $R=0$ spacetimes and self-dual Lorentzian wormholes", Phys. Rev. D 65, 064004 (2002).

[28] V. P. Frolov and I. D. Novikov, "Wormhole as a device for studying a black hole interior", Phys. Rev. D 48, 1607 (1993). 
[29] P. F. González-Días, "Ringholes and closed timelike curves", Phys. Rev. D 54, 6122 (1996).

[30] G. Clément, "Flat wormholes from cosmic strings", J. Math. Phys. 38, 5807 (1997).

[31] R. O. Aros and N. Zamorano, "Wormhole at the core of an infinite cosmic string", Phys. Rev. D 56, 6607 (1997).

[32] F. Shein, P. C. Aichelburg and W. Israel, "String-supported wormhole spacetimes containing closed timelike curves", Phys. Rev. D 54, 3800 (1996).

[33] E. Teo, "Rotating traversable wormholes", Phys. Rev. D 58, 024014 (1998).

[34] S. Nojiri, O. Obregon, S. D. Odintsov and K. E. Osetrin, "Can primordial wormholes be induced by GUTs at the early Universe?", Phys. Lett. B458, 19 (1999).

[35] A. Saa, "Nonexistence theorems for traversable wormholes", Mod. Phys. Lett. A 14, 843 (1999).

[36] S. Krasnikov, "Traversable wormhole", Phys. Rev. D 62, 084028 (2002).

[37] S. A. Hayward "Wormholes supported by pure ghost radiation", Phys. Rev. D 65, 124016 (2002).

[38] L. Á. Gergely, "Wormholes, naked singularities, and universes of ghost radiation", Phys. Rev. D 65, 127502 (2002).

[39] K. Bronnikov and S. Grinyok, "Charged wormholes with non-minimally coupled scalar fields. Existence and stability", gr-qc/0205131.

[40] P. K. Kuhfittig, "A wormhole with a special shape function", Am. J. Phys. 67, 125 (1999).

[41] D. Hochberg and M. Visser, "Null energy condition in dynamic wormholes", Phys. Rev. Lett. 81, 746 (1998). 
[42] D. Hochberg and M. Visser, "Dynamic wormholes, antitrapped surfaces, and energy conditions", Phys. Rev. D 58, 044021 (1998).

[43] M. Visser, S. Kar and N. Dadhich, "Traversable wormholes with arbitrarily small energy condition violations", Phys. Rev. Lett. 90, 201102 (2003).

[44] S. Kim, "Schwarzschild-de Sitter type wormhole", Phys. Lett. A 166, 13 (1992).

[45] T. A. Roman, "Inflating Lorentzian wormholes", Phys. Rev. D 47, 1370 (1993).

[46] M. S. R. Delgaty and R. B. Mann, "Traversable wormholes in $(2+1)$ and $(3+1)$ dimensions with a cosmological constant", Int. J. Mod. Phys. D 4, 231 (1995).

[47] A. DeBenedictis and A. Das, "On a general class of wormholes", Class. Quantum Grav. 18, 1187 (2001).

[48] A. Chodos and S. Detweiler, "Spherical symmetric solutions in fivedimensional general relativity", Gen. Rel. Grav. 14, 879 (1982).

[49] G. Clément, "A class of wormhole solutions to higher-dimensional general relativity", Gen. Rel. Grav. 16, 131 (1984).

[50] A. DeBenedictis and A. Das, "Higher dimensional wormhole geometries with compact dimensions", gr-qc/0207077.

[51] K. K. Nandi, B. Bhattacharjee, S. M. K. Alam and J. Evans, "BransDicke wormholes in the Jordan and Einstein frames", Phys. Rev. D 57, 823 (1998).

[52] Y.-G. Shen, H.-Y. Guo, Z.-Q. Tan and H.-G. Ding, "Wormholes in Kaluza-Klein theory", Phys. Rev. D 44, 1330 (1991).

[53] S. Kar, "Evolving Lorentzian wormholes", Phys. Rev. D 53, 722 (1996).

[54] L. A. Anchordoqui and S. E. Perez Bergliaffa, "Wormhole surgery and cosmology on the brane: The world is not enough", Phys. Rev. D 62, 076502 (2000). 
[55] C. Barceló and M. Visser, "Brane surgery: energy conditions, traversable wormholes, and voids", Nucl. Phys. B584, 415 (2000).

[56] H. Koyama, S. A. Hayward and S. Kim, "Construction and enlargement of dilatonic wormholes by impulsive radiation", gr-qc/0212106.

[57] M. Visser, "Traversable wormholes from surgically modified Schwarzschild spacetimes", Nucl. Phys. B328, 203 (1989).

[58] E. Poisson and M. Visser, "Thin-shell wormholes: Linearization stability", Phys. Rev. D 52, 7318 (1995).

[59] M. Ishak and K. Lake, "Stability of transparent spherically symmetric thin shells and wormholes", Phys. Rev. D 65, 044011 (2002).

[60] C. Armendáriz-Picón, "On a class of stable, traversable Lorentzian wormholes in classical general relativity". Phys. Rev. D 65, 104010 (2002).

[61] H. Shinkai and S. A. Hayward, "Fate of the first traversable wormhole: Black hole collapse or inflationary expansion", Phys. Rev. D 66, 044005 (2002).

[62] K. Bronnikov and S. Grinyok, "Instability of wormholes with a nonminimally coupled scalar field", gr-qc/0201083.

[63] M. S. Morris, K. S. Thorne and U. Yurtsever, "Wormholes, Time Machines and the Weak Energy Condition", Phy. Rev. Lett. 61, 1446 (1988).

[64] V. P. Frolov and I. D. Novikov, "Physical effects in wormholes and time machines", Phys. Rev. D 42, 1057 (1990).

[65] S. Kim and K. S. Thorne, "Do vacuum fluctuations prevent the creation of closed timelike curves?" Phys. Rev. D 43, 3929 (1991).

[66] S. W. Hawking, "Chronology protection conjecture", Phys. Rev. D 46, 603 (1992).

[67] M. Lyutikov, "Vacuum polarization at the chronology horizon of the Roman spacetime", Phys. Rev. D 49, 4041 (1994). 
[68] W. A. Hiscock and D. A. Konlowski, "Quantum vacuum energy in TaubNUT-type cosmologies", Phys. Rev. D 26, 1225 (1982).

[69] L.-X. Li and J. R. Gott, "Self-consistent vacuum for Misner space and the chronology protection conjecture", Phys. Rev. Lett. 80, 2980 (1998).

[70] W. A. Hiscock, "Quantized fields and chronology protection", grqc/0009061.

[71] M. Visser, "The quantum physics of chronology protection", grqc/0204022.

[72] K. S. Thorne, "Closed timelike curves", in General Relativity and Gravitation, Proceedings of the 13th Conference on General Relativity and Gravitation, edited by R. J. Gleiser et al (Institute of Physics Publishing, Bristol, 1993), p. 295.

[73] S. A. Hayward, "Black holes and traversible wormholes: a synthesis", gr-qc/0203051.

[74] J. G. Cramer, R. L. Forward, M. S. Morris, M. Visser, G. Benford and G. A. Landis, "Natural wormholes as gravitational lenses", Phys. Rev. D 51, 3117 (1995).

[75] D. F. Torres, G. E. Romero and L. A. Anchordoqui, "Wormholes, gamma ray bursts and the amount of negative mass in the Universe", Mod. Phys. Lett. A 13, 1575 (1998).

[76] M. Safonova, D. F. Torres and G. E. Romero, "Microlensing by natural wormholes: theory and simulations", Phys. Rev. D 65, 023001 (2002).

[77] M. Safonova, D. F. Torres, G. E. Romero, "Macrolensing signatures of large-scale violations of the weak energy condition", Mod. Phys. Lett. A 16, 153 (2001).

[78] J. P. S. Lemos, "Three-dimensional black holes and cylindrical General Relativity", Phys. Lett. B352, 46 (1995).

[79] J. P. S. Lemos and V. T. Zanchin, "Charged Rotating Black Strings and Three Dimensional Black Holes", Phys. Rev. D 54, 3840 (1996). 
[80] J. P. S. Lemos, "Rotating toroidal black holes in anti-de Sitter spacetimes and their properties", in Astronomy and Astrophysics, Recent Developments, Proceedings of the Xth Portuguese Meeting on Astronomy and Astrophysics, edited by J. P. S. Lemos et al (World Scientific, 2001), p. 88, gr-qc/001092.

[81] C. W. Misner, K. S. Thorne and J. A. Wheeler, Gravitation (W. H. Freeman and Company, New York, 1973).

[82] W. Israel, "Singular hypersurfaces and thin shells in general relativity", Nuovo Cimento B 44, 1 (1966); Nuovo Cimento B 49, 463 (1967).

[83] A. Papapetrou and A. Hamoui, "Couches simple de matière en relativité générale", Ann. Inst. Henri Poincaré A 9, 179 (1968).

[84] A. Taub, "Spacetimes with distribution valued curvature tensors", J. Math. Phys. 21, 1423 (1980). 